\title{
OPTICAL SPECTROSCOPY OF THE SURFACE POPULATION OF THE $\rho$ OPHIUCHI MOLECULAR CLOUD: THE FIRST WAVE OF STAR FORMATION
}

\author{
BRUCE A. WILKING \\ Department of Physics and Astronomy, University of Missouri-St. Louis, 1 University Boulevard, St. Louis, MO 63121; bwilking@umsl.edu
}

Michael R. Meyer

Steward Observatory, University of Arizona, Tucson, AZ 85721; mmeyer@gould.as.arizona.edu

JOHN G. ROBINSON

Department of Physics and Astronomy, University of Missouri-St. Louis, 1 University Boulevard, St. Louis, MO 63121; johnr@newton.umsl.edu

AND

Thomas P. GReENe

NASA Ames Research Center, MS 245-6, Moffett Field, CA 94035-1000; tgreene@mail.arc.nasa.gov Received 2004 December 14; accepted 2005 May 31

\begin{abstract}
We present the results of optical spectroscopy of 139 stars obtained with the Hydra multiobject spectrograph. The objects extend over a $1.3 \mathrm{deg}^{2}$ area surrounding the main cloud of the $\rho$ Oph complex. The objects were selected from narrowband images to have $\mathrm{H} \alpha$ in emission. Using the presence of strong $\mathrm{H} \alpha$ emission, lithium absorption, location in the Hertzsprung-Russell diagram, or previously reported X-ray emission, we were able to identify 88 objects as young stars associated with the cloud. Strong $\mathrm{H} \alpha$ emission was confirmed in 39 objects with line widths consistent with their origin in magnetospheric accretion columns. Two of the strongest emission-line objects are young, X-ray-emitting brown dwarf candidates with M8 spectral types. Comparisons of the bolometric luminosities and effective temperatures with theoretical models suggest a median age for this population of 2.1 Myr, which is significantly older than the ages derived for objects in the cloud core. It appears that these stars formed contemporaneously with low-mass stars in the Upper Scorpius subgroup, likely triggered by massive stars in the Upper Centaurus subgroup.
\end{abstract}

Key words: ISM: individual ( $\rho$ Ophiuchi cloud) — open clusters and associations: individual (Upper Scorpius) — stars: formation - stars: pre-main-sequence

Online material: machine-readable tables

\section{INTRODUCTION}

Nearby molecular clouds that are the sites of active star formation are observed to be surrounded by a population of less obscured and more evolved young stars. $\mathrm{H} \alpha$ emission-line and X-ray surveys have revealed large numbers of pre-mainsequence stars that extend well beyond the molecular cloud boundaries. For example, the ROSAT All-Sky Survey has led to the identification of 112 lithium-rich pre-main-sequence stars that are spread over a $450 \mathrm{deg}^{2}$ area surrounding the Orion starforming molecular clouds (Alcalá et al. 1996). While some of the young stars appear to be related to earlier star formation in the Gould Belt, at least $60 \%$ are found to be directly associated with the Orion molecular cloud complex (Alcalá et al. 2000). Proposed origins for these associated young stars include formation in rapidly moving cloudlets that have since dispersed (Feigelson 1996) or ejection from the cloud core due to threebody encounters (Sterzik \& Durisen 1995). Alternatively, they could simply be fossil tracers of previously existing dense molecular gas in analogy with OB associations and now-dispersed giant molecular clouds (e.g., Blaauw 1991). Clearly, determining the age and distribution of pre-main-sequence stars surrounding the denser molecular gas holds the key to describing the star formation history of a region and perhaps sets limits to the lifetime of the molecular cloud.

The $\rho$ Oph cloud complex, located at the edge of the Upper Scorpius subgroup in the Sco-Cen OB association, is composed of a series of filamentary dark clouds that extend eastward from cores of dense molecular gas (de Geus 1992). At a distance of about $150 \mathrm{pc}$, it is one of the closest regions of active star formation. The main cloud, L1688, hosts a $1 \mathrm{pc} \times 2$ pc centrally condensed molecular core in which the visual extinction is estimated to be 50-100 mag (Wilking \& Lada 1983). The core of L1688 has been the focus of numerous near-infrared, far-infrared, and millimeter continuum surveys, as well as X-ray and radio continuum surveys, and is found to host an embedded infrared cluster with around 200 young stellar objects (YSOs; e.g., Greene \& Young 1992; Bontemps et al. 2001; André \& Montmerle 1994; Gagné et al. 2004). Infrared spectroscopic studies infer a median age of 0.3 Myr for objects in the core (Greene \& Meyer 1995; Luhman \& Rieke 1999). It has been suggested that star formation in the $\rho$ Oph core is the latest in a chain of events that began with massive stars in the Upper Centaurus-Lupus and Lower Centaurus-Crux subgroups of the Sco-Cen OB association triggering the formation of OB stars in the Upper Scorpius subgroup, which in turn initiated star formation in the L1688 cloud (de Geus 1992).

Until recently, there has been limited study of the nature of lightly extincted association members at the periphery of the L1688 cloud that have been identified through $\mathrm{H} \alpha$ objective-prism and X-ray surveys (e.g., Dolidze \& Arakelyan 1959; Wilking et al. 1987; Montmerle et al. 1983; Casanova et al. 1995). Early spectroscopic studies of $\mathrm{H} \alpha$ emission-line stars presented spectral types for about 20 young stars (Struve 
\& Rudkjøbing 1949; Cohen \& Kuhi 1979; Rydgren 1980). An optical spectroscopic survey of $\rho$ Oph X-ray sources was conducted by Bouvier \& Appenzeller (1992) and established spectral types for 30 pre-main-sequence stars ( $23 \mathrm{new})$. They found an age of 1-10 Myr for these objects, significantly older than the age of objects in the core (see also Greene \& Meyer 1995). But these samples favored the brighter association members and gave the incorrect impression that there was a deficiency of M-type stars relative to other nearby star-forming regions (see Fig. 24 of Hillenbrand 1997). This was remedied in part by Martín et al. (1998), who obtained optical spectroscopy for 59 ROSAT-selected sources in the core and streamer of the cloud with the majority of objects classified as pre-main-sequence M stars. By examining the ratio of classical T Tauri stars (CTTSs) to weak-emission T Tauri stars (WTTSs), they found evidence for an older pre-mainsequence population outside of the L1688 core.

In an effort to identify more widely distributed and/or older late-type association members, we have obtained deep narrowband images of a $1.3 \mathrm{deg}^{2}$ area encompassing the L1688 cloud and centered at the wavelengths of $\mathrm{H} \alpha$ and [S II]. Shorter exposures at $R$ and $I$ were also obtained. This resulted in a sample of 282 candidate CTTSs with $R<20$ mag that had possible $\mathrm{H} \alpha$ emission. This sample includes association members with greater obscuration and/or lower mass than those targeted by previous surveys. Using the multiobject spectrograph Hydra, optical spectra were obtained for 129 candidate CTTSs plus 10 additional objects with $R<19.1$ mag. Our sample selection procedure and the observations are described in $\S 2$. In $\S 3$, we detail our criteria for spectral classification and our examination of surface gravities. The results of the spectral classifications and an analysis of the emission-line spectra are given in $\S 4$. Also in $\S 4$, we list the criteria used to identify 88 association members from the sample and their locations in a Hertzsprung-Russell (H-R) diagram. In $\S 5$, we compare the properties of the association members with those of low-mass stars in the Upper Scorpius subgroup of the Sco-Cen OB association to gain insight into the star formation history of the $\rho$ Oph cloud.

\section{OBSERVATIONS AND DATA REDUCTION}

Moderate-resolution spectra were obtained for 129 of 282 stars identified as candidate CTTSs from narrowband $\mathrm{H} \alpha$ images of the $\rho$ Oph cloud or from $\mathrm{H} \alpha$ objective-prism plates (Wilking et al. 1987). In addition, spectra were obtained for five bright association members and five brown dwarf candidates in the cloud not identified as candidate CTTSs. A total of 139 stars were observed. These observations are described in detail in the sections below.

\subsection{Sample Selection}

The majority of candidate CTTSs were selected from deep optical images of a $1.3 \mathrm{deg}^{2}$ area centered on the L1688 cloud obtained with the Curtis Schmidt telescope at Cerro Tololo InterAmerican Observatory. The images were obtained on 1995 March 10 , and their reduction is described elsewhere (Wilking et al. 1997). Briefly, long (45 minute) exposure narrowband images centered at the wavelengths of $\mathrm{H} \alpha(\mathrm{FWHM}=64 \AA)$ and [S II] $(\mathrm{FWHM}=45 \AA$ ) were obtained in addition to short (5 minute) exposures in the standard $R$ and $I$ bands. The latter were calibrated in the Johnson-Kron-Cousins photometric system using standard star fields established by Landolt (1992). The $R$ - and/or $I$-band photometry was obtained for over 2700 stars in the Ophiuchus field with $R<20$. Accurate positions $(<0$ "' 5$)$ for these stars were obtained using the ASTROM program distributed by the Starlink Project and a set of secondary astrometric standards and then shifted by $+0.2 \mathrm{~s}$ in right ascension to bring them into agreement with the Two Micron All Sky Survey (2MASS) coordinate system (Cutri et al. 2003). The narrowband images were used to estimate the equivalent width of possible $\mathrm{H} \alpha$ emission. The continuum in the $\mathrm{H} \alpha$ filter was estimated using the flux in the $[\mathrm{S}$ II] filter after correcting for the differences in filter widths and transmission and assuming a flat continuum with no [S II] emission. The latter assumption was shown to be valid, with detections of weak [S II] emission in only two sources. As a result, 282 stars with $R<20$ were identified with possible $\mathrm{H} \alpha$ emission $[\mathrm{EW}(\mathrm{H} \alpha)>10 \AA]$. However, because of structure in the continuum due to reddening and photospheric absorption bands, it was desirable to confirm this emission spectroscopically.

\subsection{Hydra Observations}

We obtained 151 spectra for 139 stars located toward the cloud and its periphery on 1999 May 5-6 using Hydra, the multifiber spectrograph, on the WIYN 3.5 m telescope. ${ }^{1}$ The Bench Spectrograph Camera was used with the red fiber cable, the 600 lines $\mathrm{mm}^{-1}$ grating with a blaze angle of $13^{\circ} .9$, and the GG495 filter to cover the range of 5820-8700 $\AA$ centered near $7200 \AA$. The spectral dispersion was $1.39 \AA$ pixel $^{-1}$, giving an effective resolution of $2.88 \AA$. The resolution at the central wavelength was $\lambda / \Delta \lambda=2500$. The program objects were selected by $R$ magnitude, the $\mathrm{H} \alpha$ equivalent width estimated from the narrowband images, and their accessibility to a fiber. Program objects were distributed among four fiber configurations sorted by $R$ magnitude; 12 program stars were observed in two configurations. Field 1 included the brightest candidate CTTSs $(R<15)$ and, to make most efficient use of the spectrograph, five bright X-ray sources known to be association members but with no known $\mathrm{H} \alpha$ emission. Fields 2 and 3 sampled the same magnitude range of candidate CTTSs $(15<R<17)$ with the pointing centers shifted in declination. Field 4 candidates $(17<R<19.1)$ were supplemented by five brown dwarf candidates from the study of Wilking et al. (1999, hereafter WGM99). We observed $81 \%$ of the candidate CTTSs with $R<15,88 \%$ of the candidates with $15<R<$ $17,45 \%$ of the candidates with $17<R<18$, and $21 \%$ of the candidates with $17<R<19$.1. In Table 1 we summarize the observations by presenting for each field the pointing center, $R$ magnitude range, number of candidate CTTSs observed, integration time, and number of exposures.

The spectra were reduced using the Image Reduction and Analysis Facility (IRAF). ${ }^{2}$ Images were processed for bias and dark corrections using ccdproc. Multiple exposures of a given field were median-combined and then reduced with IRAF's dohydra package. The images were flat-fielded using dome flats obtained for each fiber configuration, except for field 1, in which the flat from the configuration for field 2 was used. Sky subtraction was accomplished using the median of 7-10 sky spectra distributed across each field. The spectra were wavelength-calibrated using $5 \mathrm{~s}$ exposures of $\mathrm{CuAr}$ lamps. Scattered-light corrections were not made, and no flux calibration was performed. It was necessary in a few cases to improve the sky subtraction by first

\footnotetext{
${ }^{1}$ The WIYN Observatory is a joint facility of the University of WisconsinMadison, Indiana University, Yale University, and the National Optical Astronomy Observatory (NOAO).

${ }^{2}$ IRAF is distributed by the NOAO for the Association of Universities for Research in Astronomy (AURA), Inc.
} 
TABLE 1

Summary of Hydra Observations

\begin{tabular}{|c|c|c|c|c|c|c|c|}
\hline Field Number & $\begin{array}{l}\text { R.A. } \\
\text { (J2000.0) }\end{array}$ & $\begin{array}{c}\text { Decl. } \\
(\mathrm{J} 2000.0)\end{array}$ & $\begin{array}{c}R \\
(\mathrm{mag})\end{array}$ & Sources Observed & $\begin{array}{l}\text { Integration Time } \\
\text { (s) }\end{array}$ & Number of Exposures & $\mathrm{S} / \mathrm{N}^{\mathrm{a}}$ \\
\hline 1 & 162709.5 & -242543.5 & 15 & 28 & 600 & 4 & 21 \\
\hline 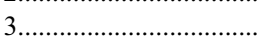 & 162719.9 & -244142.8 & $15-17$ & 35 & 1800 & 2 & 23 \\
\hline 4. & 162718.3 & -242342.9 & $17-19.1$ & 58 & 1800 & 4 & 16 \\
\hline
\end{tabular}

Noте.- Units of right ascension are hours, minutes, and seconds, and units of declination are degrees, arcminutes, and arcseconds.

${ }^{a}$ Median of S/N computed from 7400 to $7500 \AA$.

scaling the median sky spectrum before subtracting it from the source spectrum. The typical signal-to-noise ratio $(\mathrm{S} / \mathrm{N})$ for the spectra extracted in each field is presented in Table 1.

\section{ANALYSIS OF THE SPECTRA}

Spectral types were derived from visual classification (visual pattern matching of our smoothed program-star spectra with standard-star spectra) supported by quantitative analysis of some spectral indices. In this section, we provide descriptions of the absorption lines used to classify three broad groups, starting with the earliest spectral type stars (B-A), moving to the $\mathrm{F}-\mathrm{K}$ stars, and ending with the $\mathrm{M}$ stars. We conclude this section with a discussion of gravity-sensitive absorption features in the 5820 $8700 \AA$ spectral range. For the purposes of matching spectral features with those of standard stars, our Hydra spectra were smoothed using a Gaussian filter to the resolution of the standard stars for direct comparison. All spectra have been normalized to 1 by dividing out a fit to the continua, carefully excluding regions with emission lines or broad absorption due to $\mathrm{TiO}$ and $\mathrm{VO}$. Normalized spectra smoothed to a resolution of $5.7 \AA$ are shown in Figure 1 for a representative sample of program objects, with early-type stars in Figure 1 $a$ (B3-G9), K stars in Figure 1b, earlyto mid-M stars in Figure 1c, and mid- to late-M stars in Figure $1 d$. Both photospheric and telluric spectral features are labeled.

Two main sets of standards were used for classification (both qualitative and quantitative). First, optical spectra from the WIYN Hydra study of the Praesepe by Allen \& Strom (1995) were used to derive spectral types from $\mathrm{B} 8 \mathrm{~V}$ to $\mathrm{M} 4 \mathrm{~V}$. The effective resolution of these spectra was $5.7 \AA$. For giants and later type dwarfs (M5 V-M9 V), optical spectra from the study of Kirkpatrick

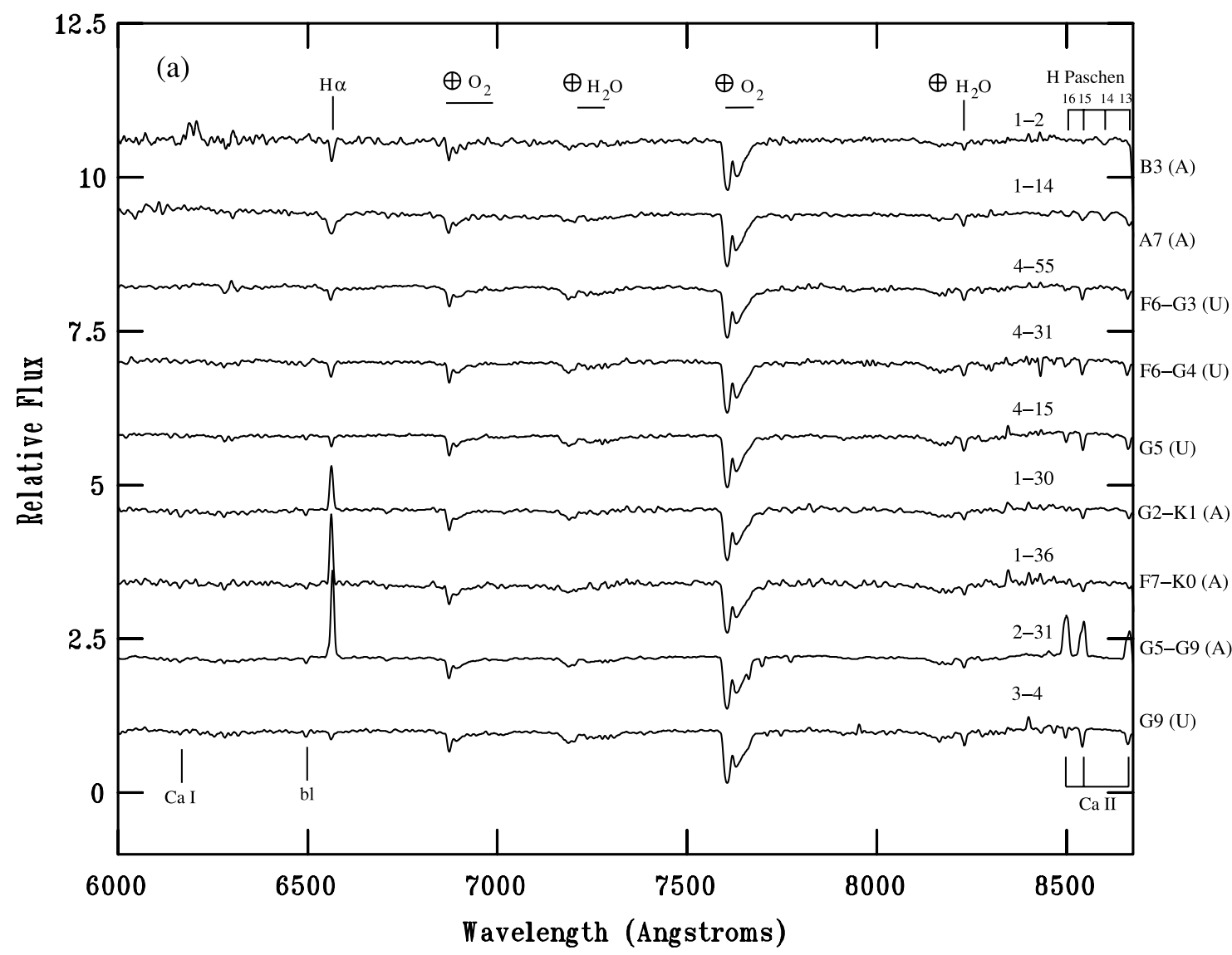

FIG. 1.-Collection of spectra representative of the entire sample, smoothed to a resolution of $5.73 \AA$ and normalized using a polynomial fit. The spectra are labeled with their field and aperture numbers (Table 2) and a spectral type or range. In addition, an indicator of association membership (A) or undetermined (U) follows the spectral type. Atomic and molecular bands of interest in our classification scheme are labeled, as are telluric features. Shown are $(a)$ B-G stars; $(b) \mathrm{K}$ stars; (c) early M stars; and $(d)$ late M stars. Included in $(c)$ and $(d)$ are spectra of giants and dwarfs from our survey to illustrate the intermediate surface gravity of the YSOs. 


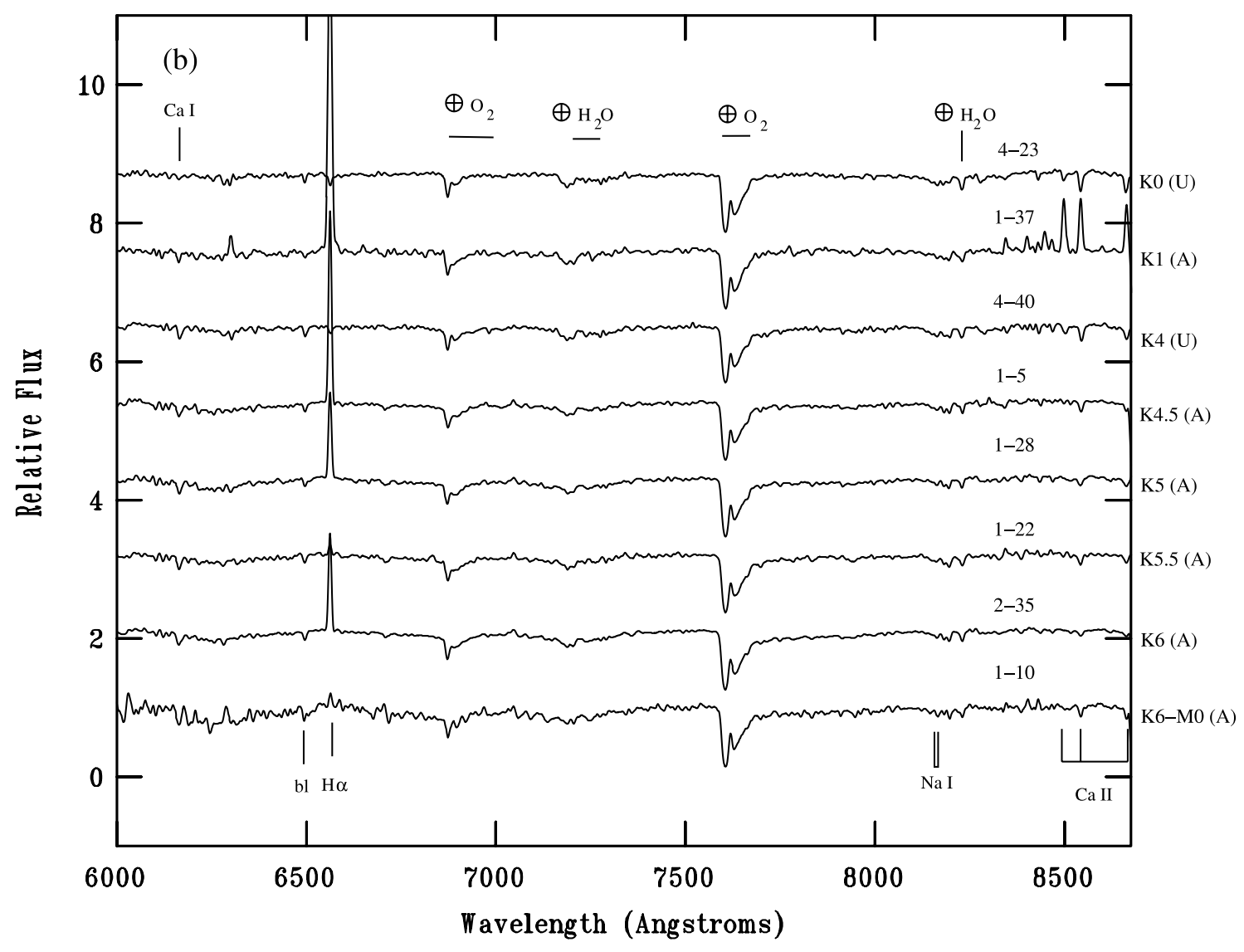

FIG. 1.-Continued

et al. (1991) were used with an effective resolution of either 8 or $18 \AA$. In addition to these, optical spectra of very late type subgiants in IC 348 (Luhman 1999) and $\rho$ Oph (Luhman et al. 1997) were used for comparison with the coolest stars in our sample. For stars earlier than B8 V, we referred to the spectral atlas of TorresDodgen \& Weaver (1993).

\subsection{Classification of $B-A$ Stars}

Absorption lines from the Balmer $(n=2)$ and Paschen $(n=3)$ series of hydrogen are prominent in the spectra of early-type stars. In our spectra, we see unblended absorption lines from $\mathrm{H} \alpha(6563 \AA)$ and Paschen $14(8598 \AA$ ) (see Fig. 1a) that reach a maximum around $\mathrm{A} 0$ and weaken in warmer stars. The $\mathrm{Ca}$ II triplet $(8498,8542,8662 \AA)$ is also observed and decreases in strength toward early-type stars until overtaken by $\mathrm{Pa} 16,15$, and $13(8502,8545$, and $8665 \AA)$. Hence, an F2 star may have an $\mathrm{EW}(\mathrm{H} \alpha)$ similar to that of a B5 star but will be distinct by displaying stronger absorption from the $\mathrm{Ca}$ II triplet. We note that all the aforementioned lines can appear in emission, and that the observed absorption-line strengths could be lower limits to the true strengths. We estimate that the uncertainty in our derived spectral types in this range is \pm 5 subclasses; however, our adopted spectral types will be greatly aided by previously published observations of these intrinsically bright stars.

\subsection{Classification of $F-K 5$ Stars}

The relative strength of absorption due to $\mathrm{H} \alpha$ and a blend of $\mathrm{Ba}$ II, $\mathrm{Fe}_{\mathrm{I}}$, and $\mathrm{Ca}$ I centered at $6497 \AA$ is the most sensitive indicator of spectral type over this range. Also helpful in spectral classification is absorption due to $\mathrm{Ca}$ I at $6122 \AA$, which grows stronger from spectral types A to K (e.g., compare Figs. $1 a$ and 1b). Absorption from the $\mathrm{Ca}$ II triplet is present but shows little variation with spectral type in $\mathrm{F}-\mathrm{K}$ stars. In general, the variation in these features is very gradual from subclass to subclass, and, when coupled with the $\mathrm{S} / \mathrm{N}$ of the spectra, the uncertainties in the spectral classifications between $\mathrm{F} 0$ and $\mathrm{K} 0$ are often $\pm 3-5$ subclasses. Paschen line absorption (13 and higher transitions) will be present through mid-F stars, but $\mathrm{Pa} 13,15$, and 16 are blended and dominated by the lines from the $\mathrm{Ca}$ II triplet at our resolution. Once again, the partial filling of $\mathrm{H} \alpha$ absorption by $\mathrm{H} \alpha$ emission in CTTSs could lead us to assign spectral types that are too late.

\subsection{Classification of K5-M9 Stars}

For stars K5 V and later, the depths of the TiO and VO bands provide the most sensitive indicators of spectral type. As shown in Figure $1 b, \mathrm{TiO}$ absorption bands begin to appear in mid-K stars centered at 6300,6700 , and $7140 \AA$ and increase in strength through M6 V stars (Figs. $1 c$ and $1 d$ ). Absorption due to TiO, which begins near the strong telluric $\mathrm{O}_{2}$ band (oxygen $B$ band; $7594-7685 \AA$ ) and extends to $7861 \AA$, is evident for spectral types of M1 V and later and is further strengthened by VO absorption from 7851 to $7973 \AA$ in mid- to late-M stars (Fig. $1 d$ ). TiO absorption bands from 8432 to $8452 \AA$ also appear for spectral types of M2 V and later and increase in strength through M9 V (Fig. 1d). For the coolest M stars (M6-M9), the depths of the $\mathrm{TiO}$ bands centered at 7140 and $7800 \AA$ appear to decrease due to the depletion of TiO into grains (e.g., Allen 1995; Fig. $2 a$ in this paper); VO absorption from 7334 to $7543 \AA$ and from 7851 to $7973 \AA$ then becomes a more reliable indicator of spectral type.

Spectral classification in this temperature range was accomplished through a combination of visual classification and quantitative analysis of $\mathrm{TiO}$ and $\mathrm{VO}$ indices, which we now describe. 


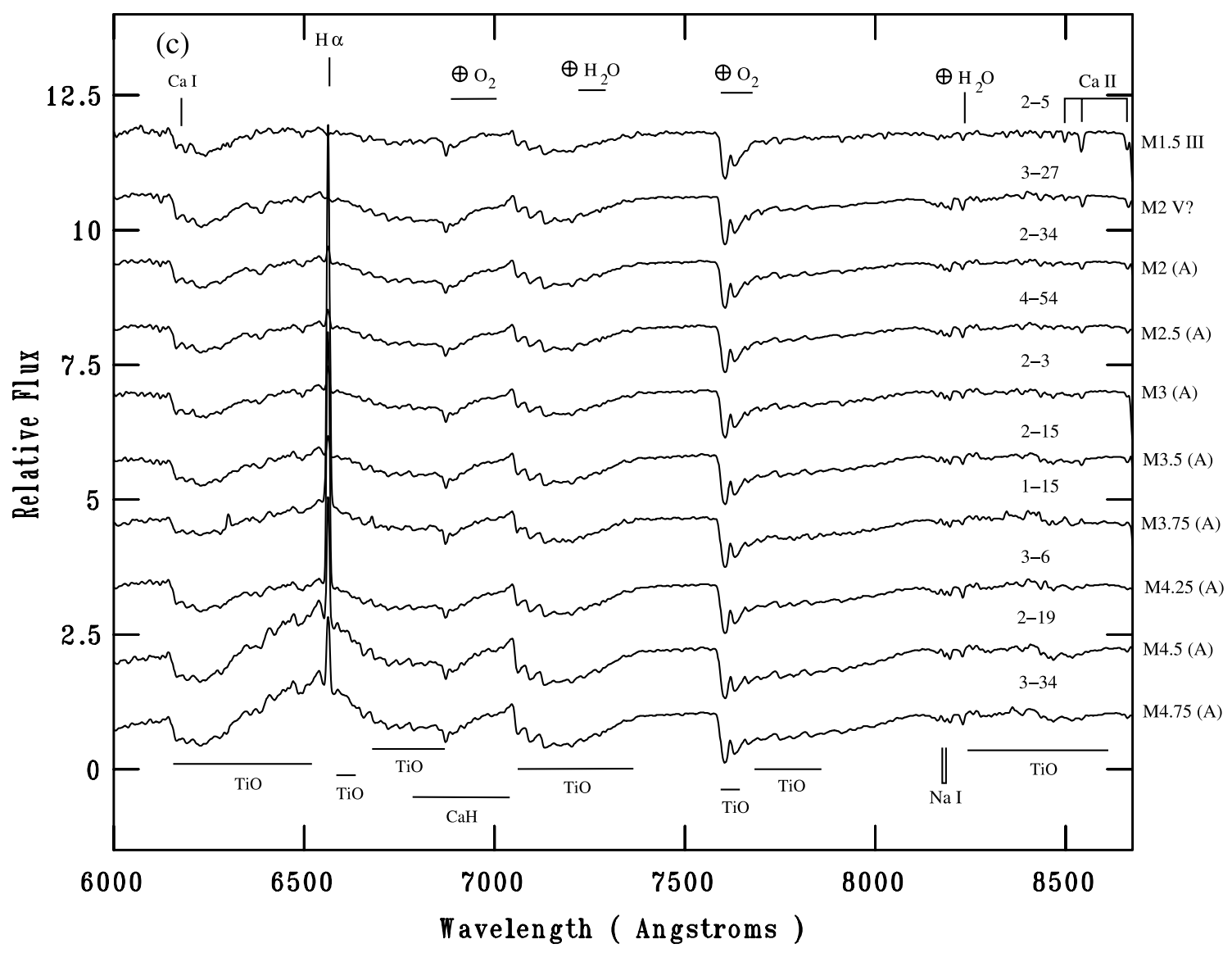

FIG. 1.-Continued

The indices were computed by taking the ratio of the average flux in a $\sim 50 \AA$ wide band of the continuum to the average in an absorption band. Following Allen (1995), we plotted the TiO index of the $7035 \AA / 7140 \AA$ ratio versus the $\mathrm{TiO}$ index of the $7500 \AA / 7800 \AA$ ratio along with their $1 \sigma$ errors for each Hydra field, as well as our dwarf standards. Figure $2 a$ shows a plot of the $\mathrm{TiO}$ indices for the dwarf standards. There is a general trend for $\mathrm{TiO}$ index to increase with later spectral type until M7, at which the trend reverses and the index decreases for stars cooler than M7. To resolve the ambiguity caused by this reversal, we developed two narrowband VO indices for spectral classification of the coolest stars in our sample. A plot of these indices is shown in Figure $2 b$ for $\mathrm{M}$ dwarf standards. The first index is the ratio of the continuum averaged over two bands that bracket the VO band centered at $7485 \AA$ to the average flux in the VO band. The second index is the ratio of the continuum in a band centered at $8120 \AA$ to the flux in the VO band centered at $7970 \AA$. As shown in Figure $2 b$, there is a general trend for the indices to increase from spectral types M3 to M9. While the use of these indices allowed us to sort objects by temperature, the final spectral classifications were made by direct comparisons of the strengths of the $\mathrm{TiO}$ and $\mathrm{VO}$ bands with standard-star spectra. The sensitivity of these absorption bands to spectral class allowed us in most cases to estimate M spectral types to within a subclass.

\subsection{Surface Gravities}

A rough estimate of the surface gravity of an object is important in determining its nature and accurately estimating its effective temperature for a given spectral type. In particular, surface gravity indicators can help identify background giants and field dwarfs that might contaminate our sample. Gravitysensitive absorption features available for analysis in our spectra include the $\mathrm{CaH}$ band centered at $6975 \AA$ and the $\mathrm{Na}$ I doublet at $8183,8195 \AA$ (both strongest in dwarfs) and absorption from the Ca II triplet at $8660 \AA$ (strongest in giants). Of these features, $\mathrm{CaH}$ is well-positioned for study.

Following Allen (1995), we have calculated a $\mathrm{CaH}$ index as the ratio of the continuum at $7035 \pm 15 \AA$ to the flux in the $\mathrm{CaH}$ absorption band at $6975 \pm 15 \AA$ and a TiO index as the ratio of the continuum at $7030 \pm 15 \AA$ to the flux in the $\mathrm{TiO}$ absorption band at $7140 \pm 15 \AA$ from the normalized spectrum of each program object. In Figure 3, we plot the $\mathrm{CaH}$ index versus the $\mathrm{TiO}$ index for 136 program objects. Error bars are computed based on the $1 \sigma$ error in flux averages and propagated to the ratios. The solid lines represent first- or second-order fits to the standard-star spectra. Based on this plot, we conclude that the surface gravities for objects with the largest $\mathrm{TiO}$ index (coolest stars) are intermediate between those of giants and dwarfs. While there are clearly late-type giants present in our sample, about $70 \%$ of the program objects have $\mathrm{TiO}$ indices $<2$ (about spectral type M4 V) and surface gravities that most closely resemble dwarf stars. Therefore, spectral types for our program objects were determined by comparing them to a grid of dwarf standards.

\section{RESULTS}

Spectral types were determined for 131 of 139 stars in the manner described in $\S 3.3$. Using the $\mathrm{CaH}$ index and other gravity-sensitive features $(\S 3.4)$, six stars were identified as giants and 26 as possible dwarfs. These data are presented in 


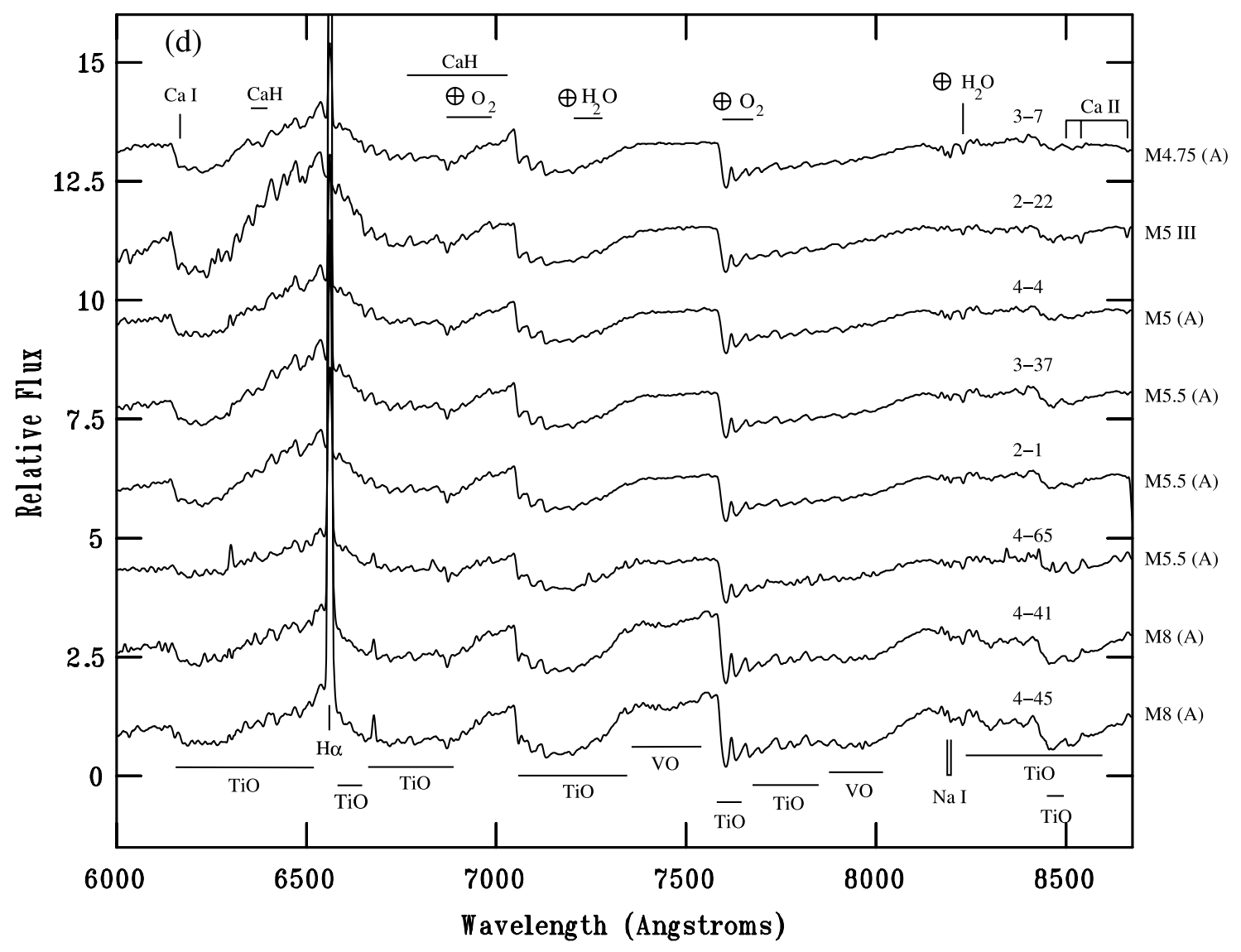

FIG. 1.-Continued
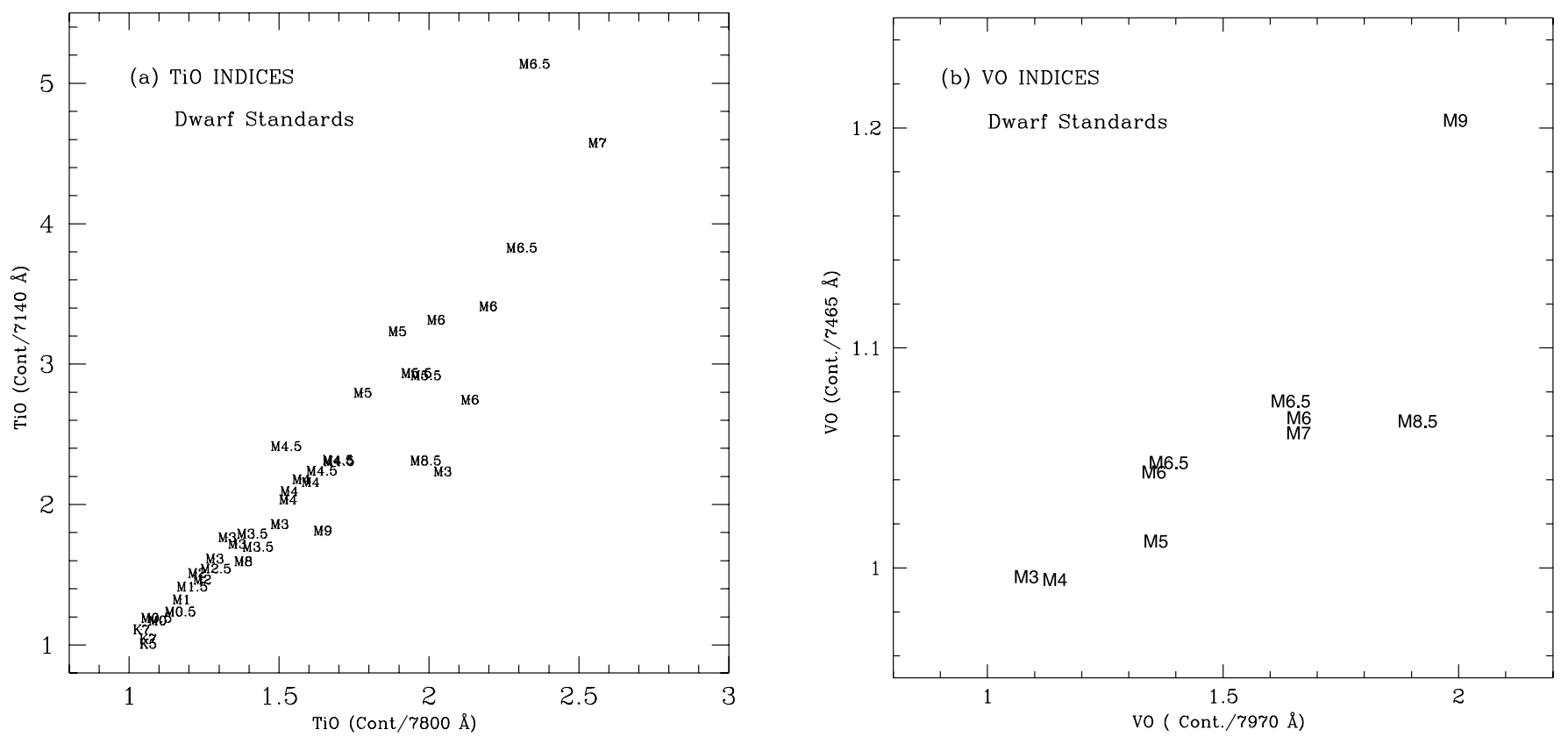

FIG. 2.- Spectral indices for dwarf standards used to guide our spectral classifications. Spectra from the studies of Allen \& Strom (1995) or Kirkpatrick et al. (1991) were used in the analysis. (a) TiO index of the $7035 \AA / 7140 \AA$ ratio vs. the TiO index of the $7500 \AA / 7800 \AA$ ratio for K5-M9 dwarfs. The ratios were formed by averaging the flux in a $30 \AA$ wide band centered on the wavelength. (b) Two VO band indices that are sensitive to spectral type for late M dwarf standard stars. The widths of the wavelength bands in the continuum were chosen to maximize the number of channels yet avoid strong absorption due to TiO. The $y$-axis shows the ratio of the continuum averaged between a $20 \AA$ wide band centered at $7380 \AA$ and a $30 \AA$ wide band centered at $7555 \AA$ to the flux in a $50 \AA$ wide band centered in the VO band at $7465 \AA$. The $x$-axis shows the ratio of the continuum in a $40 \AA$ wide band centered at $8120 \AA$ to the flux in a $60 \AA$ wide band centered in the VO band at $7970 \AA$. 


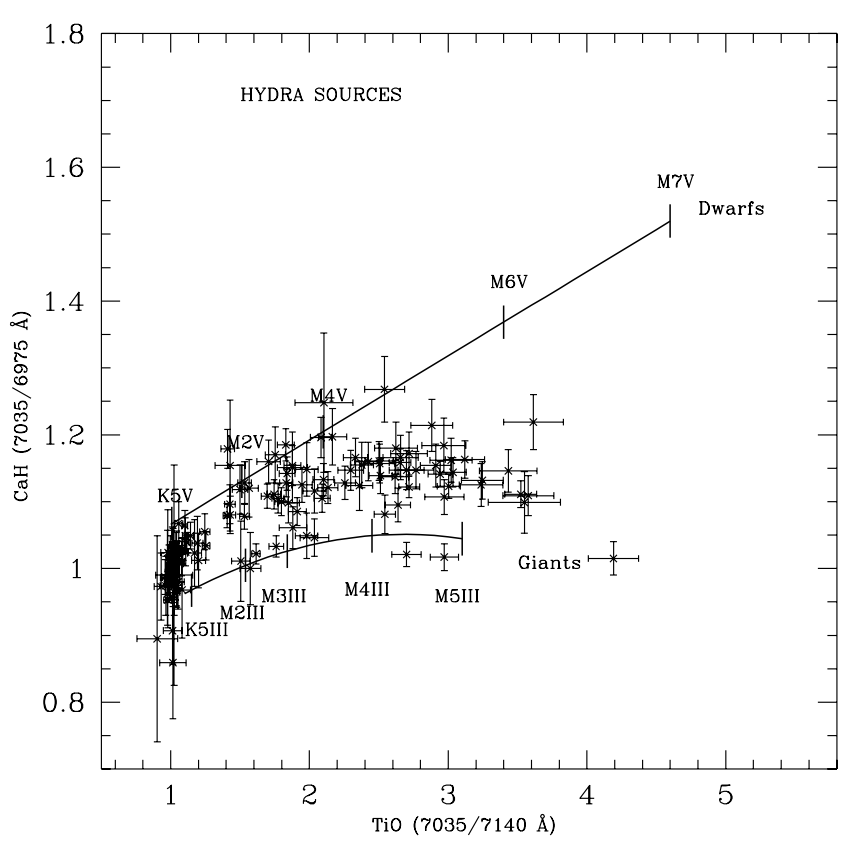

FIG. 3.- $\mathrm{CaH}$ vs. $\mathrm{TiO}$ indices as defined in the text for 136 program objects. The solid lines were derived from first- or second-order fits to dwarf and giant spectral standards. For the dwarf standards from K5 to M7, the fit was $y=$ $0.126 x+0.940$ with a correlation coefficient of $r=0.94$. We note that for spectral types later than $\mathrm{M} 7$, both indices decrease so that an $\mathrm{M} 8 \mathrm{~V}$ star has a $\mathrm{CaH}$ index similar to that of an M4 V star. For the giant standards from $\mathrm{K} 5$ to M5, the fit gave $y=-0.0357 x^{2}+0.191 x+0.795$ with a correlation coefficient of $r=0.82$.

Table 2, along with any previous source names, X-ray associations, right ascension and declination in $\mathrm{J} 2000.0$, and $R$ and I magnitudes. The derived spectral types (or range of possible spectral types) are also shown to the right of each spectrum in Figure 1 along with its classification as a possible dwarf(V?), giant (III), association member (A), or association unknown (U). The top two spectra in Figures $1 c$ and $1 d$ demonstrate the different features present in high and low surface gravity objects of the same spectral type. In the following sections, we discuss what the spectra reveal about the characteristics of the young stars and their evolutionary states. Included in this discussion is the distribution of spectral types in our sample and the variety and properties of their emission lines. A description is given of how association membership was determined and the resulting spatial distribution of association members relative to the molecular gas. Finally, the evolutionary state of the population is estimated by deriving ages and masses using an $\mathrm{H}-\mathrm{R}$ diagram.

\subsection{Distribution of Spectral Types}

The distribution of spectral types for our sample is shown in Figure 4. Over half (79) of the 131 objects with spectral-type determinations are M stars. The fact that our sample is dominated by $M$ stars is due in part to a selection effect caused by the onset of TiO absorption bands. The procedure described in $\S 2.1$ was designed to select objects with $\mathrm{H} \alpha$ emission provided the continuum was relatively flat. But the same procedure could erroneously identify $\mathrm{M}$ stars with strong $\mathrm{TiO}$ absorption as $\mathrm{H} \alpha$ emission-line objects, since the $\mathrm{H} \alpha$ filter bandpass lies in a spectral region free of $\mathrm{TiO}$ absorption, while the comparison [S II] filter bandpass lies within strong TiO absorption at $6700 \AA$.

The shaded areas of the histogram represent sources with strong $\mathrm{H} \alpha$ emission $[\mathrm{EW}(\mathrm{H} \alpha)>10 \AA]$ at the time of our observations. Our procedure of selecting $\mathrm{H} \alpha$ emission-line stars from narrowband images was only moderately successful. In particular, our procedure appears to break down for the fainter sources $(R<17)$, which accounts for the number of background $\mathrm{G}$ stars in our spectroscopic sample. Our highest success rate occurred with mid-K stars and mid- to late-M stars and was $65 \%$. In the latter group, $\mathrm{H} \alpha$ emission was clearly a factor in their identification in our original narrowband image. There is clearly no deficiency of M-type CTTSs in the $\rho$ Oph cloud relative to other nearby star-forming regions. The apparent lack of $\mathrm{M}$ stars in previous spectroscopic surveys represents the bias toward brighter stars in those surveys.

\subsection{Emission-Line Spectra}

Nearly one-third of our sample for which we could determine spectral types displayed strong emission from $\mathrm{H} \alpha$. The spectra for about one-third of these strong emitters also exhibited emission lines from $\mathrm{He}$ I, O I, Ca II, and/or Pa 14. Emission lines were identified, and their equivalent widths and FWHMs were measured using Gaussian fits. No attempt was made to deconvolve the emission profile by the instrument response. The results for all CTTSs are presented in Table 3.

Since our sample was based on a procedure designed to select sources with strong $\mathrm{H} \alpha$ emission characteristic of CTTSs, it is not surprising that 39 of the 131 objects with spectral-type determinations displayed strong $\mathrm{H} \alpha$ emission. Of these, 26 are newly identified CTTSs based on an $\mathrm{EW}(\mathrm{H} \alpha)>10 \AA$ criteria. The previously identified CTTSs can be found in Table 2 by looking at sources with previously measured $\mathrm{H} \alpha$ emission (col. [10]). An additional 17 objects showed weaker $\mathrm{H} \alpha$ emission $[10 \AA>$ $\mathrm{EW}(\mathrm{H} \alpha)>5 \AA$ ] , with nearly all having spectral types from M3 to M5. We note that variability plays a role in the identification of $\mathrm{H} \alpha$ emission (e.g., Wilking et al. 1987; Hartigan 1993). Among the six $\mathrm{H} \alpha$ emitters with multiple observations, the equivalent width of object 2-30 (field number and aperture from Table 2) dropped from 38 to $8.5 \AA$ in only 1 day. The brown dwarf candidate GY 5 was observed to have an $\operatorname{EW}(\mathrm{H} \alpha)=15 \AA$, whereas a similar spectrum obtained in 1998 showed no detectable emission (WGM99). In addition, the shape of the emission profile also appeared to change in three cases (Chini 8, EL 24, and object 2-30). All the strong $\mathrm{H} \alpha$ emission lines were well-resolved with typical FWHMs of $250 \mathrm{~km} \mathrm{~s}^{-1}$ compared to the $128 \mathrm{~km} \mathrm{~s}^{-1}$ velocity resolution of our data.

These broad $\mathrm{H} \alpha$ profiles, which can exceed $300 \mathrm{~km} \mathrm{~s}^{-1}$ in our sample, have been successfully modeled as arising in magnetospheric accretion columns (Hartmann et al. 1994; Muzerolle et al. 2001). Thirteen of the H $\alpha$ profiles were clearly asymmetric or displayed non-Gaussian wings. The profile shapes are noted in Table 3 using the classification scheme of Reipurth et al. (1996). The prevalence of wings or asymmetries on the blue side of the profile is typical among CTTSs and suggests the presence of mass outflows. The Ca II triplet at $8660 \AA$ was observed in emission in 14 of the $\mathrm{H} \alpha$ emitters and was strongest in the G- or K-type objects. No attempt was made to deblend them from the weaker Paschen emission lines $\mathrm{Pa} 13,15$, and 16. In general, the $\mathrm{Ca}$ II lines were narrower than $\mathrm{H} \alpha$ and barely resolved in our data, with typical FWHMs of $150 \mathrm{~km} \mathrm{~s}^{-1}$. Figure 5 shows an expanded view of the $\mathrm{H} \alpha$ emission and $\mathrm{Ca}$ II emission lines for a representative sample of seven CTTSs. As in Figure 1, the spectra have been smoothed to a resolution of $5.7 \AA$. H $\alpha$ profiles for objects $1-24$ and 1-29 were symmetric, while the rest of the profiles in Figure $5 a$ exhibited a blueward asymmetry. Only the source object 2-31/1-35 (Figs. $5 a$ and $5 b$, bottom two spectra) displayed asymmetric profiles in both $\mathrm{Ca}$ II and $\mathrm{H} \alpha$. 
TABLE 2

Optical Properties of Candidate T Tauri Stars

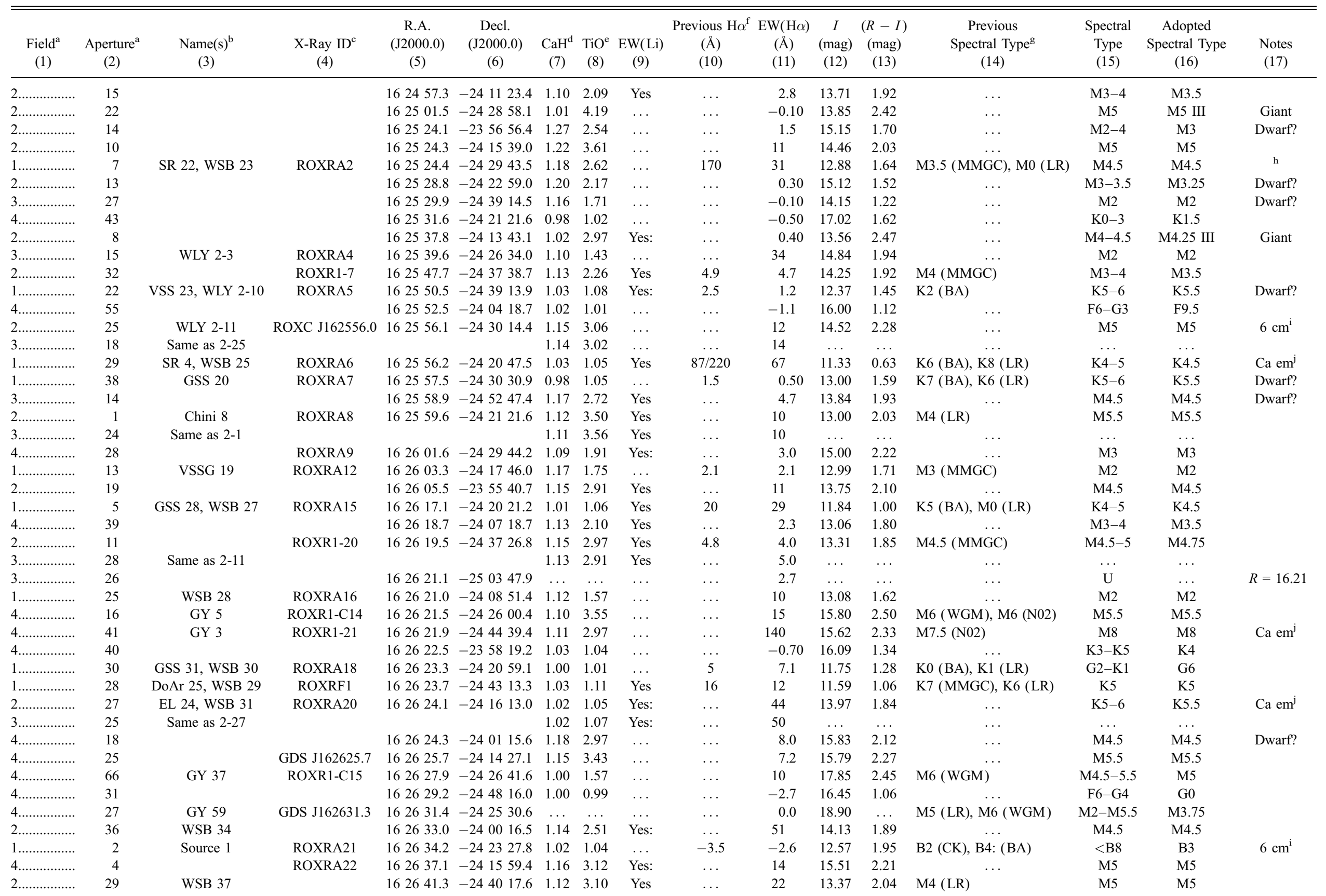


TABLE 2-Continued

\begin{tabular}{|c|c|c|c|c|c|c|c|c|c|c|c|c|c|c|c|c|}
\hline $\begin{array}{l}\text { Field }^{\mathrm{a}} \\
\text { (1) }\end{array}$ & $\begin{array}{l}\text { Aperture }^{\mathrm{a}} \\
\text { (2) }\end{array}$ & $\begin{array}{c}\operatorname{Name}(s)^{b} \\
\text { (3) }\end{array}$ & $\begin{array}{c}\text { X-Ray } I^{c} \\
\text { (4) }\end{array}$ & $\begin{array}{l}\text { R.A. } \\
(\mathrm{J} 2000.0) \\
(5)\end{array}$ & $\begin{array}{c}\text { Decl. } \\
(\mathrm{J} 2000.0) \\
(6)\end{array}$ & $\begin{array}{l}\mathrm{CaH}^{\mathrm{d}} \\
(7)\end{array}$ & $\begin{array}{c}\mathrm{TiO}^{\mathrm{e}} \\
(8)\end{array}$ & $\begin{array}{c}\mathrm{EW}(\mathrm{Li}) \\
(9)\end{array}$ & $\begin{array}{c}\text { Previous } \mathrm{H} \alpha^{\mathrm{f}} \\
(\AA \AA) \\
(10)\end{array}$ & $\begin{array}{c}\mathrm{EW}(\mathrm{H} \alpha) \\
(\AA) \\
(11)\end{array}$ & $\begin{array}{c}I \\
(\mathrm{mag}) \\
(12)\end{array}$ & $\begin{array}{c}(R-I) \\
(\mathrm{mag}) \\
(13)\end{array}$ & $\begin{array}{c}\text { Previous } \\
\text { Spectral Type } \\
\text { (14) }\end{array}$ & $\begin{array}{l}\text { Spectral } \\
\text { Type } \\
(15)\end{array}$ & $\begin{array}{c}\text { Adopted } \\
\text { Spectral Type } \\
\text { (16) }\end{array}$ & $\begin{array}{l}\text { Notes } \\
(17)\end{array}$ \\
\hline $3 \ldots \ldots \ldots \ldots \ldots$ & 3 & Same as $2-29$ & & & & 1.12 & 2.90 & Yes & $\ldots$ & 25 & $\cdots$ & . & & & $\cdots$ & \\
\hline $4 \ldots \ldots \ldots \ldots \ldots . .$. & 11 & GY 107 & & 162642.5 & $-2422 \quad 17.1$ & & & $\ldots$ & $\ldots$ & 0.0 & & & M3 III (WGM) & $\mathrm{U}$ & & \\
\hline $1 \ldots \ldots \ldots \ldots \ldots . .$. & 21 & & ROXC J162643.2 & 162643.1 & -241109.1 & 1.01 & 1.20 & $\ldots$ & $\ldots$ & 0.70 & 13.30 & 1.53 & & K7-M0 & K8 & \\
\hline $1 \ldots \ldots \ldots \ldots \ldots \ldots$ & 20 & GY 112 & ROXRF2 & 162644.3 & $-24 \quad 43 \quad 13.5$ & 1.06 & 1.88 & $\ldots$ & 7.8 & 6.0 & 13.22 & 1.75 & M3.5 (MMGC) & M2-4 & M3 & \\
\hline $3 \ldots \ldots \ldots \ldots \ldots \ldots$ & 22 & & ROXN6 & 162644.4 & $-24 \quad 4713.3$ & 1.16 & 2.51 & Yes & $\ldots$ & 3.5 & 13.88 & 1.84 & $\ldots$ & M4.5 & M4.5 & Dwarf? \\
\hline $4 \ldots \ldots \ldots \ldots \ldots .$. & 13 & & & 162646.1 & $-2358 \quad 10.1$ & 1.16 & 2.42 & Yes & $\ldots$ & 2.8 & 15.52 & 2.11 & $\ldots$ & M2-4 & M3 & \\
\hline $1 \ldots \ldots \ldots \ldots \ldots \ldots$ & 36 & WSB 38 & ROXRA24 & 162646.5 & -241159.7 & 0.97 & 1.04 & Yes & 19 & 10 & 12.37 & 1.53 & G9: (BA), K8 (LR) & F7-K0 & G3.5 & \\
\hline $4 \ldots \ldots \ldots \ldots \ldots \ldots$ & 30 & & ROXRF3 & 162647.1 & -244429.1 & 1.15 & 2.70 & Yes & $\ldots$ & 5.4 & 15.19 & 2.22 & $\ldots$ & M4.5 & M4.5 & Dwarf? \\
\hline $4 \ldots \ldots \ldots \ldots \ldots$ & 59 & & & 162648.2 & $-24 \quad 4203.2$ & 1.05 & 1.98 & $\ldots$ & $\ldots$ & 1.0 & 15.56 & 2.25 & $\ldots$ & M3-4.5 & M3.75 & \\
\hline 1 1..................... & 32 & WSB 40 & & 162648.6 & -235634.3 & 1.06 & 1.25 & $\ldots$ & $\ldots$ & 23 & 12.92 & 1.53 & $\ldots$ & K5-6 & K5.5 & $\mathrm{Ca} \mathrm{em}{ }^{\mathrm{j}}$ \\
\hline $4 \ldots \ldots \ldots \ldots \ldots . .$. & 53 & WL 18 & $\mathrm{ROXCH} 3$ & 162649.0 & -243824.8 & 0.97 & 1.08 & $\ldots$ & $\ldots$ & 96 & 17.38 & 1.95 & $\ldots$ & K5-K8 & K6.5 & $\mathrm{Ca} \mathrm{em}{ }^{\mathrm{i}}$ \\
\hline $2 \ldots \ldots \ldots \ldots \ldots$ & 38 & & & 162650.5 & -241351.9 & 1.16 & 2.50 & $\ldots$ & $\ldots$ & 5.5 & 14.78 & 2.04 & $\ldots$ & M4.5 & M4.5 & Dwarf? \\
\hline $2 \ldots \ldots \ldots \ldots \ldots$ & 17 & & & 162651.7 & -240353.9 & 1.02 & 2.70 & $\ldots$ & $\ldots$ & 0.10 & 13.11 & 2.36 & $\ldots$ & M4 & M4 III & Giant \\
\hline $4 \ldots \ldots \ldots \ldots \ldots . . .$. & 32 & & & 162653.2 & -240558.2 & 1.02 & 1.00 & $\ldots$ & $\ldots$ & -1.9 & 16.88 & 1.52 & $\ldots$ & G9-K2 & K0.5 & \\
\hline $2 \ldots \ldots \ldots \ldots \ldots$ & 9 & & & 162655.0 & -241016.5 & 1.20 & 2.08 & $\ldots$ & $\ldots$ & -0.40 & 13.81 & 1.47 & $\ldots$ & M2-4 & M3 & Dwarf? \\
\hline $4 \ldots \ldots \ldots \ldots \ldots$ & 46 & & ROXC J162656.7 & 162656.7 & -241351.2 & 1.02 & 1.17 & $\ldots$ & $\ldots$ & 14 & 16.29 & 2.17 & $\ldots$ & K5-M0 & K7 & \\
\hline $4 \ldots \ldots \ldots \ldots \ldots \ldots$ & 26 & & & 162657.8 & -245237.1 & 0.98 & 1.04 & $\ldots$ & $\ldots$ & -0.20 & 17.45 & 1.01 & $\ldots$ & K5 & K5 & Dwarf? \\
\hline $1 \ldots \ldots \ldots \ldots \ldots \ldots$ & 37 & SR 24s, WSB 42 & ROXRF7 & 162658.5 & -244536.4 & 1.00 & 1.04 & $\ldots$ & 76 & 50 & 12.87 & 1.28 & K7 (MMGC), K2 (LR) & K0-2 & K1 & $\mathrm{Ca} \mathrm{em}{ }^{\mathrm{j}}$ \\
\hline $4 \ldots \ldots \ldots \ldots \ldots \ldots$ & 42 & & & 162659.1 & -235639.0 & 0.99 & 0.98 & $\ldots$ & $\ldots$ & 0.50 & 15.95 & 1.26 & $\ldots$ & U & $\ldots$ & \\
\hline $4 \ldots \ldots \ldots \ldots \ldots .$. & 63 & & & 162706.6 & -240702.9 & 1.15 & 2.77 & $\ldots$ & $\ldots$ & 5.7 & 15.34 & 2.19 & $\ldots$ & M4.5 & M4.5 & Dwarf? \\
\hline $4 \ldots \ldots \ldots \ldots \ldots . .$. & 65 & GY 204 & ROXCH17 & 162706.7 & -244148.8 & 1.08 & 2.54 & $\ldots$ & $\ldots$ & 43 & 15.43 & 2.14 & M6 (N02) & M5.5 & M5.5 & $\mathrm{Ca} \mathrm{em}{ }^{\mathrm{j}}$ \\
\hline $4 \ldots \ldots \ldots \ldots \ldots . .$. & 37 & & & 162709.1 & -241200.6 & 1.10 & 1.85 & $\ldots$ & $\ldots$ & 4.0 & 16.14 & 2.33 & $\cdots$ & M2-3 & M2.5 & \\
\hline $3 \ldots \ldots \ldots \ldots \ldots$ & 44 & ROX 20a, WSB 45 & ROXN27 & 162714.5 & -245133.4 & 1.17 & 2.33 & Yes & 3 & 3.6 & 13.31 & 1.65 & M5 (BA), M1-4 (LR) & M4.5 & M4.5 & Dwarf? \\
\hline $1 \ldots \ldots \ldots \ldots \ldots . .$. & 9 & ROX 20b, WSB 46 & ROXRF14 & 162715.1 & -245138.5 & 1.13 & 1.53 & Yes: & 6.3 & 5.0 & 12.26 & 1.26 & M2 (BA), M0-3 (LR) & M2 & M2 & Dwarf? \\
\hline 3.................... & 17 & WSB 47 & & 162717.0 & -244710.9 & 1.01 & 1.08 & $\ldots$ & $\ldots$ & -1.1 & 15.06 & 1.78 & $\ldots$ & K0-2 & K1 & \\
\hline 4................. & 10 & & & 162717.5 & -240513.4 & 1.08 & 1.53 & $\ldots$ & .. & 95 & 15.47 & 2.20 & .. & M3-4 & M3.5 & $\mathrm{Ca} \mathrm{em}{ }^{\mathrm{j}}$ \\
\hline $4 \ldots \ldots \ldots \ldots \ldots$ & 9 & & & 162718.1 & $-2453 \quad 16.5$ & 1.15 & 1.88 & $\ldots$ & $\ldots$ & 1.0 & 16.63 & 2.02 & $\ldots$ & M2-3 & M2.5 & Dwarf? \\
\hline $1 \ldots \ldots \ldots \ldots \ldots$ & 6 & WSB 48 & & 162718.4 & -245452.5 & 1.25 & 2.10 & $\ldots$ & $\ldots$ & 90 & 13.23 & 1.47 & $\ldots$ & M3-4.5 & M3.75 & \\
\hline 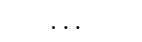 & $\ldots$ & SR 12 & $\mathrm{ROXCH} 40$ & 162719.7 & -244139.9 & . & $\cdots$ & $\ldots$ & $4.5 / 8.8$ & $\ldots$ & 11.10 & 1.30 & K4/M2.5 (BA), M0 (LR) & $\ldots$ & M0 & $6 \mathrm{~cm}^{\mathrm{i}}$ \\
\hline $4 \ldots \ldots \ldots \ldots \ldots . .$. & 62 & & & 162720.4 & -235842.5 & 0.91 & 1.01 & $\ldots$ & $\ldots$ & -0.20 & 17.42 & 1.31 & $\ldots$ & $\mathrm{U}$ & $\ldots$ & \\
\hline $3 \ldots \ldots \ldots \ldots \ldots$ & 6 & WSB 49 & ROXRF19 & 162723.0 & -244806.8 & 1.12 & 2.13 & Yes & $\ldots$ & 37 & 13.31 & 1.75 & $\ldots$ & M4-4.5 & M4.25 & \\
\hline $4 \ldots \ldots \ldots \ldots \ldots$ & 45 & GY 264 & ROXCH52 & 162726.6 & -242554.2 & 1.12 & 3.24 & $\ldots$ & $\ldots$ & 155 & 15.72 & 2.18 & $\ldots$ & M8 & M8 & $\mathrm{Ca} \mathrm{em}{ }^{\mathrm{j}}$ \\
\hline $3 \ldots \ldots \ldots \ldots \ldots \ldots$ & 7 & & ROXRF24 & 162728.8 & -245431.4 & 1.21 & 2.88 & $\ldots$ & $\ldots$ & 8.0 & 14.32 & 1.70 & $\ldots$ & M4.5-5 & M4.75 & Dwarf? \\
\hline $2 \ldots \ldots \ldots \ldots \ldots$ & 20 & & ROXR1-48 & 162730.5 & -243234.7 & 1.19 & 1.83 & $\ldots$ & $\ldots$ & -0.10 & 14.30 & 1.34 & $\ldots$ & M2 & M2 & Dwarf? \\
\hline $3 \ldots \ldots \ldots \ldots \ldots$ & 33 & Same as $2-20$ & & & & 1.18 & 1.83 & $\ldots$ & $\ldots$ & -0.20 & & & 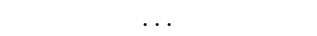 & & & \\
\hline $4 \ldots \ldots \ldots \ldots \ldots . . .$. & 22 & GY 284 & ROXR1-C27 & 162730.9 & -242456.1 & 1.12 & 2.04 & $\ldots$ & $\ldots$ & 2.9 & 16.24 & 2.21 & M1.5 (LR) & M3-3.5 & M3.25 & \\
\hline $4 \ldots \ldots \ldots \ldots \ldots . .$. & 12 & & & 162731.0 & -240344.1 & 0.99 & 1.02 & $\ldots$ & $\ldots$ & -0.30 & 17.63 & 1.13 & $\ldots$ & $\mathrm{U}$ & $\ldots$ & \\
\hline $1 \ldots \ldots \ldots \ldots \ldots$ & 19 & & & 162731.3 & -235908.7 & 1.18 & 1.41 & $\ldots$ & $\ldots$ & 0.10 & 13.12 & 1.02 & $\ldots$ & M1 & M1 & Dwarf? \\
\hline $2 \ldots \ldots \ldots \ldots \ldots$ & 6 & GY 295, WSB 50 & ROXCH70 & 162735.3 & -243833.4 & 1.14 & 2.77 & Yes & $\ldots$ & 4.2 & 14.08 & 2.08 & M4 (LR) & M4.5 & M4.5 & \\
\hline 3............... & 41 & Same as $2-6$ & & & & 1.11 & 2.66 & Yes & $\ldots$ & 4.0 & & .. & & & .. & \\
\hline $1 \ldots \ldots \ldots \ldots \ldots . .$. & 1 & GY 297 & & 162736.5 & -242833.3 & 1.12 & 1.50 & $\ldots$ & $\ldots$ & -0.27 & 13.30 & 1.37 & M0-3 (LR) & M2 & M2 & Dwarf? \\
\hline $4 \ldots \ldots \ldots \ldots \ldots$ & 54 & & & 162738.0 & -235724.1 & 1.11 & 1.74 & Yes & .. & 3.0 & 13.71 & 1.74 & & M2-3 & M2.5 & \\
\hline $2 \ldots \ldots \ldots \ldots \ldots$ & 35 & DoAr 32, WSB 51 & ROX 30b & 162738.3 & -235732.7 & 1.04 & 1.12 & Yes & 22 & 13 & 12.30 & 1.36 & K4 (BA) & K5-7 & K6 & \\
\hline $4 \ldots \ldots \ldots \ldots \ldots$ & 24 & WLY 2-49 & ROXRF29 & 162738.3 & -243658.4 & 1.05 & 1.11 & $\ldots$ & $\ldots$ & 20 & 15.52 & 2.17 & K8 (LR) & $\mathrm{K} 5-6$ & K5.5 & \\
\hline 4.................... & 38 & GY 310 & ROXCH76 & 162738.6 & -243838.4 & 0.79 & 1.70 & $\cdots$ & $\cdots$ & 0.0 & 17.40 & $\ldots$ & M6 (N02), M8.5 (WGM) & M3-5 & M4 & \\
\hline $1 \ldots \ldots \ldots \ldots \ldots$ & 31 & WSB 53 & & 162739.0 & -235818.9 & 1.06 & 1.10 & Yes: & 14 & 18 & 12.02 & 1.22 & K4 (BA) & K5-6 & K5.5 & \\
\hline $3 \ldots \ldots \ldots \ldots \ldots$ & 5 & GY 314, WSB 52 & ROXRF30 & 162739.5 & -243915.6 & 1.05 & 1.14 & Yes & 30 & 39 & 14.03 & 1.73 & M0 (LR, MMGC) & K5 & K5 & $\mathrm{Ca} \mathrm{em},{ }^{\mathrm{j}} 6 \mathrm{~cm}^{\mathrm{i}}$ \\
\hline
\end{tabular}


TABLE 2-Continued

\begin{tabular}{|c|c|c|c|c|c|c|c|c|c|c|c|c|c|c|c|c|}
\hline $\begin{array}{l}\text { Field }^{\mathrm{a}} \\
\text { (1) }\end{array}$ & $\begin{array}{l}\text { Aperture }^{\mathrm{a}} \\
\text { (2) }\end{array}$ & $\begin{array}{c}\text { Name(s) } \\
\text { (3) }\end{array}$ & $\begin{array}{c}\text { X-Ray ID } \\
\text { (4) }\end{array}$ & $\begin{array}{c}\text { R.A. } \\
(\mathrm{J} 2000.0) \\
(5)\end{array}$ & $\begin{array}{c}\text { Decl. } \\
(\mathrm{J} 2000.0) \\
(6)\end{array}$ & $\begin{array}{c}\mathrm{CaH}^{\mathrm{d}} \\
\text { (7) }\end{array}$ & $\begin{array}{c}\mathrm{TiO}^{\mathrm{e}} \\
(8)\end{array}$ & $\begin{array}{c}\mathrm{EW}(\mathrm{Li}) \\
(9)\end{array}$ & $\begin{array}{c}\text { Previous } H \alpha^{\mathrm{f}} \\
(\AA \AA) \\
(10)\end{array}$ & $\begin{array}{c}\mathrm{EW}(\mathrm{H} \alpha) \\
(\AA) \\
(11)\end{array}$ & $\begin{array}{c}I \\
(\mathrm{mag}) \\
(12)\end{array}$ & $\begin{array}{c}(R-I) \\
(\mathrm{mag}) \\
(13)\end{array}$ & $\begin{array}{c}\text { Previous } \\
\text { Spectral Type } \\
\text { (14) }\end{array}$ & $\begin{array}{l}\text { Spectral } \\
\text { Type } \\
(15)\end{array}$ & $\begin{array}{c}\text { Adopted } \\
\text { Spectral Type } \\
\text { (16) }\end{array}$ & $\begin{array}{l}\text { Notes } \\
(17)\end{array}$ \\
\hline $1 \ldots \ldots \ldots \ldots$ & 26 & SR 9, WSB 54 & ROXRF31 & 162740.3 & -242204.1 & 1.03 & 1.09 & Yes & $10.5 / 14$ & 23 & 11.26 & 0.50 & K5 (BA), K8 (LR) & K5 & K5 & \\
\hline $2 \ldots \ldots \ldots \ldots$ & 34 & & ROXC J162741.8 & 162741.8 & -240427.3 & 1.13 & 1.83 & Yes: & $\ldots$ & 2.5 & 13.39 & 1.82 & & M2 & M2 & Dwarf? \\
\hline 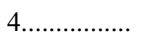 & 64 & GY 326 & ROXCH81 & 162742.7 & -243851.0 & 1.01 & 1.51 & $\ldots$ & $\ldots$ & 0.20 & 17.30 & $\ldots$ & M4 (WGM) & M2 & M2 & \\
\hline $3 \ldots \ldots \ldots \ldots$ & 21 & & & 162745.2 & $\begin{array}{lll}-25 & 03 & 32.7\end{array}$ & 1.17 & 2.66 & Yes & $\ldots$ & 3.5 & 14.09 & 1.83 & $\ldots$ & M4.5 & M4.5 & Dwarf? \\
\hline $4 \ldots \ldots \ldots \ldots \ldots$ & 47 & & & 162749.3 & -235609.0 & 1.02 & 1.01 & $\ldots$ & $\ldots$ & 0.0 & 16.48 & 1.06 & $\ldots$ & F6-G9 & $\mathrm{G} 2.5$ & \\
\hline $1 \ldots \ldots \ldots \ldots$ & 14 & VSSG 14 & ROXR1-53 & 162749.9 & -242540.3 & 0.98 & 0.98 & $\ldots$ & -9.1 & -8.0 & 12.19 & 1.50 & A7 (GM, MMGC) & $<\mathrm{F} 1$ & A7 & $6 \mathrm{~cm}^{\mathrm{i}}$ \\
\hline $1 \ldots \ldots \ldots \ldots$ & 10 & ROX 31 & ROXRF33 & 162752.1 & -244050.3 & 1.04 & 1.19 & & 3.3 & 1.3 & 12.66 & 1.60 & K7 (BA) & K6-M0 & K7.5 & $6 \mathrm{~cm}^{\mathrm{i}}$ \\
\hline $1 \ldots \ldots \ldots \ldots$ & 24 & SR 10, WSB 57 & ROXR1-C12 & 162755.6 & -242618.0 & 1.11 & 1.70 & Yes: & 43 & 56 & 11.95 & 1.18 & M1.5 (CK), M0 (LR) & M2 & M2 & $\mathrm{Ca} \mathrm{em}{ }^{\mathrm{j}}$ \\
\hline $4 \ldots \ldots \ldots \ldots \ldots$ & 8 & & & 162759.7 & -235716.5 & 1.15 & 1.43 & & $\ldots$ & 0.60 & 16.99 & 1.76 & $\ldots$ & M0 & M0 & \\
\hline $3 \ldots \ldots \ldots \ldots$ & 34 & WSB 58 & ROXRF34 & 162800.0 & -244819.3 & 1.14 & 2.62 & Yes: & $\ldots$ & 7.5 & 13.40 & 1.98 & $\ldots$ & M4.5-5 & M4.75 & \\
\hline $3 \ldots \ldots \ldots \ldots$ & 31 & & ROXRF35 & 162800.1 & -245342.5 & 1.13 & 3.25 & Yes & $\ldots$ & 14 & 13.51 & 2.05 & $\ldots$ & M5 & M5 & \\
\hline $2 \ldots \ldots \ldots \ldots \ldots$ & 3 & & & 162800.8 & -240052.0 & 1.10 & 1.77 & Yes & $\ldots$ & 3.2 & 13.89 & 1.85 & $\ldots$ & $\mathrm{M} 2-4$ & M3 & \\
\hline $4 \ldots \ldots \ldots \ldots . . .$. & 61 & & & 162804.9 & -235608.4 & 0.97 & 0.97 & $\ldots$ & $\ldots$ & -0.10 & 17.26 & 1.23 & $\ldots$ & K5 & K5 & \\
\hline $4 \ldots \ldots \ldots \ldots \ldots \ldots$ & 5 & & & 162809.9 & -244847.5 & 1.00 & 1.03 & $\ldots$ & $\ldots$ & -0.80 & 16.66 & 0.88 & $\ldots$ & K4-5 & K4.5 & \\
\hline $2 \ldots \ldots \ldots \ldots \ldots$ & 21 & & & 162811.1 & -240617.9 & 1.16 & 2.38 & Yes: & $\ldots$ & 7.7 & 14.27 & 2.13 & $\ldots$ & M3-4.5 & M3.75 & \\
\hline 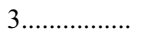 & 13 & & & 162811.3 & -245905.8 & 1.02 & 0.99 & .. & $\ldots$ & -1.5 & 15.90 & 1.00 & $\ldots$ & G5-G9 & G7 & \\
\hline $3 \ldots \ldots \ldots \ldots \ldots$ & 11 & & ROXRF36 & 162812.4 & -245044.9 & 1.15 & 1.88 & Yes & $\ldots$ & 6.0 & 13.67 & 1.93 & $\ldots$ & M2-4 & M3 & Dwarf? \\
\hline $4 \ldots \ldots \ldots \ldots \ldots \ldots$ & 51 & & & 162814.4 & -235752.1 & 1.01 & 1.01 & $\ldots$ & $\ldots$ & 0.0 & 17.49 & 1.18 & $\ldots$ & G5-9 & G7 & \\
\hline $3 \ldots \ldots \ldots \ldots$ & 9 & & & 162815.1 & -250207.3 & 1.03 & 1.02 & $\ldots$ & $\ldots$ & -1.5 & 15.32 & 0.92 & $\ldots$ & G9-K0 & G9.5 & \\
\hline $3 \ldots \ldots \ldots \ldots$ & 19 & & & 162815.6 & -250349.5 & 1.00 & 1.02 & & $\ldots$ & -0.40 & 15.53 & 0.47 & $\ldots$ & G9-K1 & K0 & \\
\hline $3 \ldots \ldots \ldots \ldots$ & 20 & WSB 60 & & 162816.6 & -243658.3 & 1.09 & 2.64 & Yes: & $\ldots$ & 81 & 14.46 & 2.09 & K8 (LR) & M4.5 & M4.5 & $\mathrm{Ca} \mathrm{em}^{\mathrm{j}}$ \\
\hline $4 \ldots \ldots \ldots \ldots$ & 58 & & & 162816.6 & -240736.4 & 1.07 & 1.06 & $\ldots$ & $\ldots$ & 0.0 & 16.77 & 1.69 & $\ldots$ & $\mathrm{U}$ & $\ldots$ & \\
\hline $4 \ldots \ldots \ldots \ldots$ & 14 & & & 162818.1 & -235730.4 & 1.00 & 1.01 & $\ldots$ & $\ldots$ & -1.5 & 16.57 & 1.08 & $\ldots$ & F6-G4 & G0 & \\
\hline $3 \ldots \ldots \ldots \ldots \ldots \ldots$ & 12 & & & 162819.2 & -245734.2 & 1.15 & 1.98 & Yes: & $\ldots$ & 4.9 & 13.54 & 1.67 & $\ldots$ & M2-4 & M3 & Dwarf? \\
\hline $2 \ldots \ldots \ldots \ldots$ & 12 & & & 162820.0 & -242610.9 & 0.97 & 0.93 & $\ldots$ & $\ldots$ & -0.80 & 15.69 & 1.21 & $\ldots$ & F9-G9 & G5 & \\
\hline $4 \ldots \ldots \ldots \ldots \ldots . .$. & 3 & & & 162820.1 & -242317.8 & 1.15 & 2.30 & Yes: & $\ldots$ & 4.1 & 15.55 & 2.14 & $\ldots$ & M3-4 & M3.5 & \\
\hline $2 \ldots \ldots \ldots \ldots \ldots \ldots$ & 37 & & ROXC J162821.5 & 162821.6 & -242155.2 & 1.14 & 1.48 & $\ldots$ & $\ldots$ & 1.0 & 14.95 & 2.02 & $\ldots$ & M2-3 & M2.5 & \\
\hline 3...................... & 35 & Same as $2-37$ & & & & 1.14 & 1.84 & Yes: & $\ldots$ & 3.0 & $\ldots$ & $\ldots$ & $\ldots$ & $\ldots$ & $\ldots$ & \\
\hline $2 \ldots \ldots \ldots \ldots$ & 5 & & & 162823.3 & -241834.3 & 1.03 & 1.59 & $\ldots$ & $\ldots$ & -0.80 & 14.38 & 2.04 & $\ldots$ & M1-2 & M1.5 III & Giant \\
\hline $3 \ldots \ldots \ldots \ldots$ & 29 & Same as $2-5$ & & & & 1.02 & 1.65 & $\ldots$ & $\ldots$ & -1.0 & $\ldots$ & $\ldots$ & $\ldots$ & $\ldots$ & $\ldots$ & \\
\hline $2 \ldots \ldots \ldots \ldots$ & 30 & & ROXC J162823.4 & 162823.4 & -242240.6 & 1.03 & 1.02 & $\ldots$ & $\ldots$ & 35 & 14.60 & 1.69 & $\ldots$ & K5 & K5 & $\operatorname{Var} \mathrm{H} \alpha$ \\
\hline $3 \ldots \ldots \ldots \ldots$ & 42 & Same as $2-30$ & & & & 1.02 & 1.05 & Yes: & $\ldots$ & 8.5 & $\ldots$ & & $\ldots$ & $\ldots$ & $\ldots$ & \\
\hline $2 \ldots \ldots \ldots \ldots \ldots$ & 4 & & ROXC J162824.2 & 162824.3 & -240931.9 & 1.08 & 1.43 & & $\ldots$ & 2.4 & 14.60 & 1.92 & $\ldots$ & M2 & M2 & Dwarf? \\
\hline $3 \ldots \ldots \ldots \ldots$ & 39 & & & 162824.9 & -243543.4 & 1.16 & 3.02 & Yes & $\ldots$ & 6.0 & 14.79 & 1.99 & $\ldots$ & M5 & M5 & \\
\hline $1 \ldots \ldots \ldots . . . . . .$. & 11 & & & 162825.1 & -242820.1 & 1.05 & 2.04 & $\ldots$ & $\ldots$ & -0.50 & 12.46 & 1.79 & $\ldots$ & M2 & M2 III & Giant \\
\hline $4 \ldots \ldots \ldots \ldots . .$. & 44 & & & 162828.3 & -235851.0 & 0.90 & 0.90 & $\ldots$ & $\ldots$ & -0.20 & 17.17 & 1.20 & $\ldots$ & $\mathrm{U}$ & $\ldots$ & \\
\hline $3 \ldots \ldots \ldots \ldots . .$. & 2 & & & 162828.3 & -245221.6 & 1.03 & 1.26 & $\ldots$ & $\ldots$ & -0.80 & 15.05 & 1.35 & $\ldots$ & K6 & K6 & \\
\hline $4 \ldots \ldots \ldots \ldots . . .$. & 23 & & & 162829.4 & -243120.9 & 0.99 & 0.99 & $\ldots$ & $\ldots$ & -0.90 & 16.56 & 1.22 & $\ldots$ & K0 & K0 & \\
\hline $4 \ldots \ldots \ldots \ldots . . .$. & 57 & & & 162832.6 & -241524.1 & 1.12 & 2.36 & & $\ldots$ & 4.0 & 15.95 & 2.35 & $\ldots$ & M3-4 & M3.5 & \\
\hline 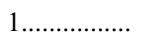 & 35 & SR 20, WSB 61 & ROX 33 & 162832.7 & -242245.2 & 1.00 & 1.00 & Yes: & 21 & 15 & 11.36 & 1.23 & G0: (BA), K8 (LR) & G5-9 & G7 & $\mathrm{db} \mathrm{Ca} \mathrm{em}{ }^{\mathrm{j}}$ \\
\hline $2 \ldots \ldots \ldots \ldots$ & 31 & Same as $1-35$ & & & & 1.00 & 1.00 & Yes: & $\ldots$ & 15 & $\ldots$ & $\ldots$ & $\ldots$ & $\ldots$ & $\ldots$ & \\
\hline $4 \ldots \ldots \ldots \ldots \ldots$ & 50 & & & 162836.3 & -240043.2 & 0.99 & 1.00 & $\ldots$ & $\ldots$ & -1.2 & 16.28 & 1.57 & $\ldots$ & $\mathrm{K} 0-2$ & K1 & \\
\hline $2 \ldots \ldots \ldots \ldots \ldots$ & 23 & & ROXC J162843.1 & 162843.1 & -242252.3 & 1.16 & 2.62 & $\ldots$ & $\ldots$ & 6.4 & 14.47 & 2.07 & $\ldots$ & M4.5-5 & M4.75 & $\mathrm{k}$ \\
\hline 3...................... & 1 & Same as $2-23$ & & & & 1.16 & 2.69 & Yes & $\ldots$ & 6.0 & $\ldots$ & $\ldots$ & $\ldots$ & $\ldots$ & $\ldots$ & \\
\hline $4 \ldots \ldots \ldots \ldots \ldots$ & 1 & & & 162843.6 & -240444.7 & 1.00 & 0.99 & .. & $\ldots$ & -2.5 & 15.75 & 1.27 & $\ldots$ & F7-G3 & G0 & \\
\hline $1 \ldots \ldots \ldots \ldots$ & 15 & SR 13, WSB 62 & ROX 34 & 162845.3 & -242819.0 & 1.10 & 1.80 & Yes: & $30 / 48$ & 46 & 10.95 & 1.45 & $\mathrm{M} 2.5(\mathrm{BA})$ & M3-4.5 & M3.75 & $\mathrm{Ca} \mathrm{em}{ }^{\mathrm{j}}$ \\
\hline 4..................... & 49 & & & 162847.0 & -243252.6 & 0.98 & 1.00 & $\ldots$ & $\ldots$ & -1.20 & 16.85 & 1.10 & $\ldots$ & G5-9 & G7 & \\
\hline
\end{tabular}


TABLE 2-Continued

\begin{tabular}{|c|c|c|c|c|c|c|c|c|c|c|c|c|c|c|c|c|}
\hline $\begin{array}{l}\text { Field }^{\mathrm{a}} \\
\text { (1) }\end{array}$ & $\begin{array}{l}\text { Aperture }^{\mathrm{a}} \\
\text { (2) }\end{array}$ & $\begin{array}{l}\text { Name(s) } \\
\text { (3) }\end{array}$ & $\begin{array}{l}\text { X-Ray } I^{c}{ }^{c} \\
\text { (4) }\end{array}$ & $\begin{array}{c}\text { R.A. } \\
(\mathrm{J} 2000.0) \\
(5)\end{array}$ & $\begin{array}{c}\text { Decl. } \\
(\mathrm{J} 2000.0) \\
(6)\end{array}$ & $\begin{array}{c}\mathrm{CaH}^{\mathrm{d}} \\
\text { (7) }\end{array}$ & $\begin{array}{l}\mathrm{TiO}^{\mathrm{e}} \\
(8)\end{array}$ & $\begin{array}{c}\mathrm{EW}(\mathrm{Li}) \\
(9)\end{array}$ & $\begin{array}{c}\text { Previous } \mathrm{H} \alpha^{\mathrm{f}} \\
(\AA) \\
(10)\end{array}$ & $\begin{array}{c}\mathrm{EW}(\mathrm{H} \alpha) \\
(\AA) \\
(11)\end{array}$ & $\begin{array}{c}I \\
(\mathrm{mag}) \\
(12)\end{array}$ & $\begin{array}{c}(R-I) \\
(\mathrm{mag}) \\
(13)\end{array}$ & $\begin{array}{c}\text { Previous } \\
\text { Spectral Type } \\
\text { (14) }\end{array}$ & $\begin{array}{l}\text { Spectral } \\
\text { Type } \\
(15)\end{array}$ & $\begin{array}{c}\text { Adopted } \\
\text { Spectral Type } \\
\text { (16) }\end{array}$ & $\begin{array}{l}\text { Notes } \\
(17)\end{array}$ \\
\hline $3 \ldots \ldots \ldots \ldots . . . .$. & 43 & WSB 63 & ROXC J162854.0 & 162854.1 & -244744.7 & 1.08 & 1.41 & Yes & $\ldots$ & 50 & 13.54 & 1.70 & $\ldots$ & M1-2 & M1.5 & \\
\hline $4 \ldots \ldots$ & 56 & & & 162859.2 & -240515.6 & 0.95 & 0.98 & $\ldots$ & $\ldots$ & -1.9 & 17.04 & 0.78 & $\ldots$ & F6-G9 & G2.5 & \\
\hline 3.................... & 4 & & & 162900.0 & -242639.2 & 1.01 & 1.00 & $\ldots$ & $\ldots$ & -1.1 & 15.20 & 1.00 & $\ldots$ & G9 & G9 & \\
\hline 4 & 52 & & & 162902.0 & -243110.7 & 0.98 & 0.99 & $\ldots$ & $\ldots$ & -0.90 & 17.39 & 0.91 & $\ldots$ & G5-K0 & G7.5 & \\
\hline 4 & 35 & & & 162903.8 & -24302.4 & 1.13 & 1.95 & $\ldots$ & $\ldots$ & -0.50 & 16.54 & 1.71 & $\ldots$ & M2-4 & M3 & Dwarf? \\
\hline 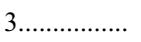 & 37 & & & 162904.0 & -245142.0 & 1.11 & 3.58 & Yes & $\ldots$ & 9.0 & 14.49 & 2.04 & $\ldots$ & M5.5 & M5.5 & \\
\hline $1 \ldots \ldots \ldots \ldots \ldots$ & 3 & & & 162912.7 & -242355.8 & 1.03 & 1.76 & $\ldots$ & $\ldots$ & -1.1 & 11.68 & $\ldots$ & $\ldots$ & M1-2 & M1.5 III & Giant \\
\hline $4 \ldots \ldots$ & 60 & & & 162921.1 & -242132.2 & 0.86 & 1.02 & $\ldots$ & $\ldots$ & -0.10 & 17.70 & 0.96 & $\ldots$ & $\mathrm{U}$ & $\ldots$ & \\
\hline $4 \ldots \ldots$ & 21 & & & 162923.5 & -242751.2 & 0.98 & 0.99 & $\ldots$ & $\ldots$ & -1.4 & 17.48 & 0.87 & $\ldots$ & K0-2 & K1 & \\
\hline $4 \ldots \ldots \ldots \ldots \ldots . .$. & 15 & & & 162923.9 & -242910.8 & 0.99 & 0.98 & $\ldots$ & $\ldots$ & -1.4 & 16.11 & 1.02 & $\ldots$ & G5 & G5 & \\
\hline 4 & 2 & & & 162924.5 & -243054.8 & 0.96 & 1.03 & $\ldots$ & $\ldots$ & 1.0 & 17.67 & 0.87 & $\ldots$ & F6-G9 & $\mathrm{G} 2.5$ & \\
\hline $4 \ldots \ldots \ldots \ldots \ldots .$. & 36 & & & 162927.7 & -242439.0 & 0.99 & 0.96 & $\ldots$ & $\ldots$ & -1.5 & 17.45 & 0.78 & $\ldots$ & F6-G0 & F8 & \\
\hline
\end{tabular}

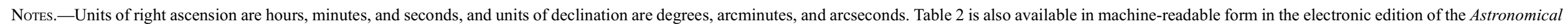
Journal.

${ }^{a}$ Hydra field and aperture number of observation.

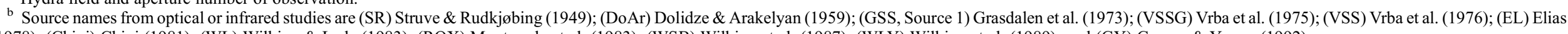
(1978); (Chini) Chini (1981); (WL) Wilking \& Lada (1983); (ROX) Montmerle et al. (1983); (WSB) Wilking et al. (1987); (WLY) Wilking et al. (1989); and (GY) Greene \& Young (1992).

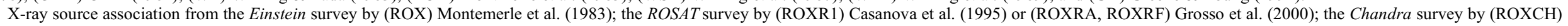
Imanishi et al. (2001), (GDS) Gagné et al. (2004), or (ROXC) N. Grosso et al. (2005, in preparation); or observations with XMM-Newton by (ROXN) Ozawa et al. (2005).

${ }^{\mathrm{d}} \mathrm{CaH}$ index calculated from the ratio of flux from the center of the continuum at $7035 \AA$ to the center of the $\mathrm{CaH}$ absorption at $6975 \AA$.

e $\mathrm{TiO}$ index calculated from the ratio of flux from the center of the continuum at $7035 \AA$ to the center of the TiO band at $7140 \AA$.

Previously reported equivalent width of $\mathrm{H} \alpha$ by Cohen \& Kuhi (1979), Bouvier \& Appenzeller (1992), or Martín et al. (1998).

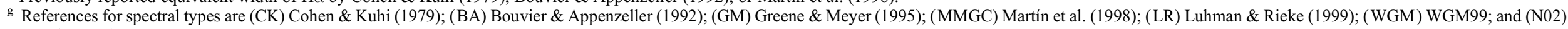
Natta et al. (2002).

$R$ - and $I$-band photometry from S. Gordon \& K. Strom (1990, unpublished).

Radio continuum source at $\lambda=6 \mathrm{~cm}$ observed by Andre et al. (1987), Leous et al. (1991), or Gagné et al. (2004).

"Ca em" and " $\mathrm{db} \mathrm{Ca}$ em" refer to $\mathrm{Ca}$ emission and double $\mathrm{Ca}$ emission, respectively.

${ }^{\mathrm{k}} \mathrm{S} / \mathrm{N}$ in $6700 \AA$ region is too low to detect Li absorption. 


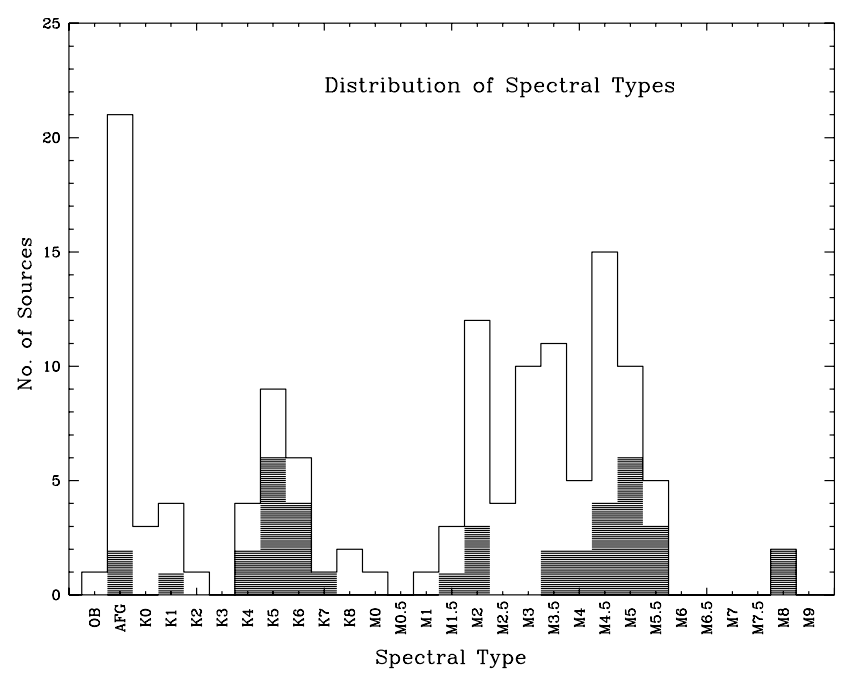

FIG. 4.-Distribution of spectral types derived for 131 program objects. The bins were chosen to permit a direct comparison with similar histograms derived for other star-forming regions (e.g., Hillenbrand 1997). The shaded areas indicate objects that displayed strong $\mathrm{H} \alpha$ emission $[\mathrm{EW}(\mathrm{H} \alpha)>10 \AA]$.

Emission lines from both permitted and forbidden atomic transitions were detected in the spectra of the strongest $\mathrm{H} \alpha$ emitters in Table 3 with $\mathrm{EW}(\mathrm{H} \alpha)>28 \AA$. Both $\mathrm{Pa} 14$ and $\mathrm{O}_{\mathrm{I}}$ (7773 $\AA$ ) displayed broad profiles with FWHMs similar to that of $\mathrm{H} \alpha$. The other $\mathrm{O}$ I lines and the He I lines all appeared to be barely resolved, with FWHMs between 150 and $180 \mathrm{~km} \mathrm{~s}^{-1}$. The detection of forbidden-line emission from [O I ] was observed in six objects. Weak [S II] emission at $6731 \AA$ was detected in only two objects (SR 22 and WSB 46). The detection of these lines is consistent with the presence of winds associated with CTTSs (Edwards et al. 1987).

\subsection{Identification of Pre-Main-Sequence Association Members}

Pre-main-sequence objects that are products of recent star formation in the Ophiuchus region were identified using one or more of the following four criteria. First, candidates with $\mathrm{H} \alpha$ emission with EW $>10 \AA$ resemble CTTSs, a property exhibited by 39 stars in our study (see Table 2, col. [11]). Second, association with X-ray emission is a signpost of youth and has been observed in 61 stars from our sample by various surveys of the region (see Table 2, col. [4]). Third, the presence of lithium absorption is an indicator of youth. While the lithium absorption was not well resolved in our spectra, it was definitely detected in 30 stars (Table 2, col. [9]). Finally, 58 objects (excluding giants identified in Table 2) that are too luminous to be main-sequence objects at the distance to $\rho$ Oph and have an estimate for $A_{v}$ too high to be foreground to the cloud $\left(A_{v}>\right.$ $1.5 \mathrm{mag}$ ) are identified as pre-main-sequence objects. Table 4 lists the 88 association members identified by our observations followed by the criteria used in their classification. At least 25 of these objects are newly identified association members. The bright association member SR 12 has been included in the list; while we did not obtain a spectrum, it was present in our $R$ - and $I$-band images and had a previously well-determined spectral type.

We note that of the 26 objects with dwarflike surface gravities (Table 2), 19 are identified as association members based on the second, third, or fourth criterion. None display strong $\mathrm{H} \alpha$ emission. Without performing a quantitative analysis, the strengths of their $\mathrm{CaH}$ and $\mathrm{Na}$ I absorption lines are indistinguishable from those of main-sequence stars.

\subsubsection{Lithium}

The presence of $\mathrm{Li}$ I absorption at $6707 \AA$ in stellar spectra indicates that the objects have cool interiors $\left(<2 \times 10^{6} \mathrm{~K}\right)$ and are young. Measurements proved to be difficult for most objects due to our spectral resolution and neighboring iron lines. The line was definitely observed in 30 stars with higher $\mathrm{S} / \mathrm{N}$ spectra ("yes" in Table 2, col. [9]) and tentatively detected in 19 stars ("yes:" in Table 2, col. [9]). Four stars were identified as young objects solely on the basis of lithium absorption (Table 4), and all were located above the main sequence in the H-R diagram. The absence of a Li entry in Tables 2 and 4 does not imply that $\mathrm{Li}$ I is not present, only that the feature was not discernible in our spectra.

\subsubsection{Brown Dwarfs}

This program included seven brown dwarf candidates identified through low-resolution infrared spectroscopy: GY 3, GY 5, GY 37, GY 59, GY 204, GY 310, and GY 326 (WGM99; Luhman \& Rieke 1999; Natta et al. 2002). The new optical spectral types agreed with the infrared classifications within two subclasses for six out of seven sources. Using infrared spectra, GY 310 was classified as M8.5 by WGM99 and B. A. Wilking et al. (2005, in preparation) and as M6 by Natta et al. (2002). While the spectrum is noisy, our optical classification of M4 for GY 310 is significantly earlier and, if confirmed, may suggest the presence of an infrared companion.

The two coolest objects in our sample, 4-41 (GY 3) and 4-45 (GY 264), are classified with M8 spectral types. Their spectra, shown in Figure 1d, feature strong VO absorption at 73347534 and 7851-7973 $\AA$. The shorter wavelength VO forms a obvious depression in the continuum between two TiO bands. The longer wavelength $\mathrm{VO}$ broadens the absorption from the 7666-7861 TiO band starting around $7900 \AA$. Their classification as young brown dwarf candidates was confirmed by their strong $\mathrm{H} \alpha$ emission $[\mathrm{EW}(\mathrm{H} \alpha)=140$ and $155 \AA$, respectively], association with X-ray emission (see Table 2), and location in the H-R diagram (§ 4.4). Object 4-41, also known as ISO Oph 32, was first identified as a low-mass object by Natta et al. (2002). Excess emission at $K\left(r_{k}=0.34\right)$ and in the mid-infrared (Bontemps et al. 2001) are indicative of a circumstellar disk (Natta et al. 2002). ${ }^{3}$ Object 4-45 is a previously unidentified brown dwarf candidate. It was previously misidentified as a foreground $\mathrm{M}$ star based on VRI photometry (ophmd5; Festin 1998). It does not appear to have an infrared excess with $r_{k}=0.02$. The object lies in close proximity to the marginal Herbig-Haro candidate P2 (Phelps \& Barsony 2004).

\subsubsection{Distribution of Association Members}

The distribution of the 88 association members identified by this study is shown in Figure 6 relative to contours of ${ }^{13} \mathrm{CO}$ column density that delineate the cloud boundaries. We note that due to the high extinction in the cloud core, our data sample only the surface population toward the densest gas. For reference, star symbols mark the locations of the Sco-Cen B star $\rho$ Oph A and the most massive member of the L 1688 embedded cluster, Oph S1. There is a definite tendency for association members to concentrate near the highest column density gas in

\footnotetext{
${ }^{3}$ Using $J H K$ photometry from the 2MASS catalog converted into the California Institute of Technology photometric system, $r_{k}=F_{K, \text { ex }} / F_{K}=\left[\left(1+r_{h}\right)\right.$ $\left.\left(10^{\left[(H-K)-(H-K)_{0}-0.065 A_{v}\right] / 2.5}\right)\right]-1$, where $r_{h}=F_{H, \mathrm{ex}} / F_{H}=\left(10^{\left[(J-H)-(J-H)_{0}-0.11 A_{v}\right] / 2.5}\right)$ -1 , with intrinsic colors and visual extinctions computed for an M8 spectral type.
} 
TABLE 3

Gaussian Fits to Emission Lines of T Tauri Stars

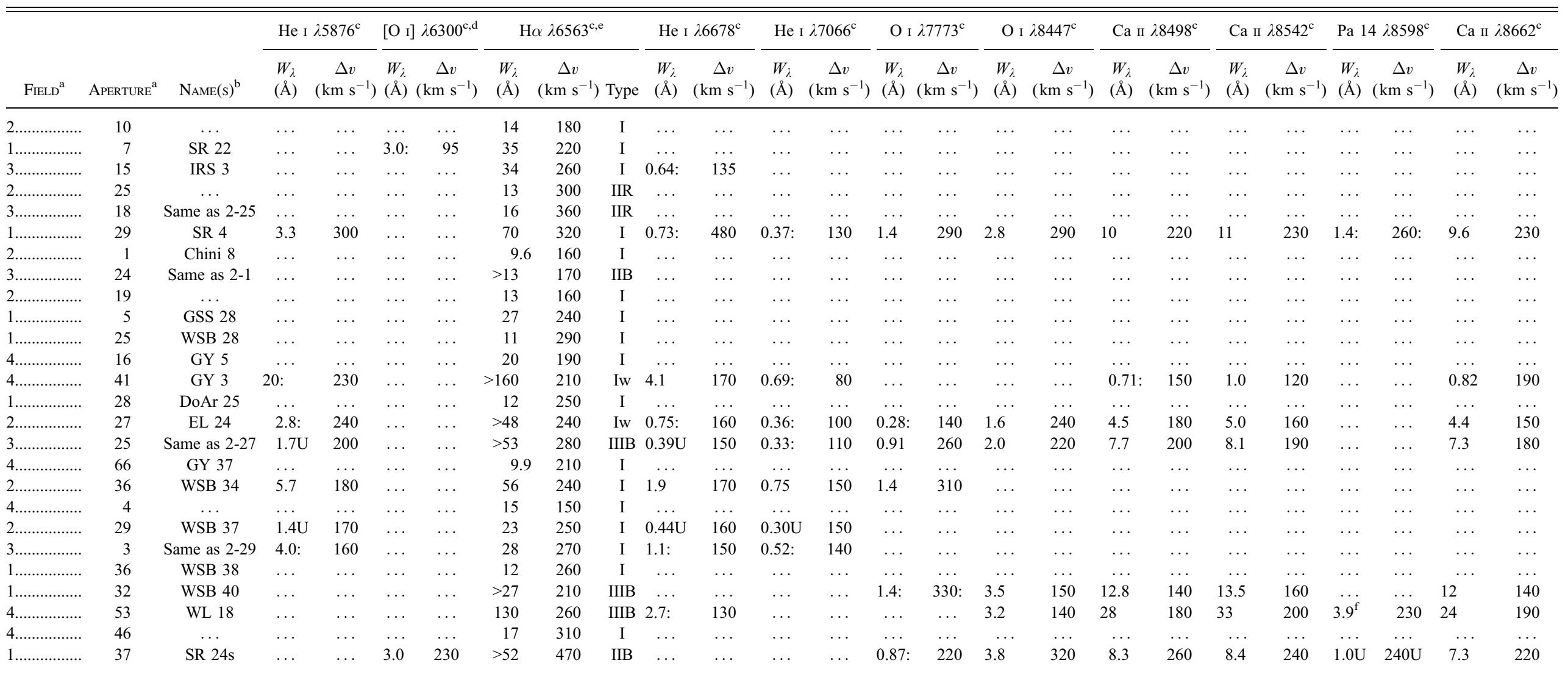


TABLE 3-Continued

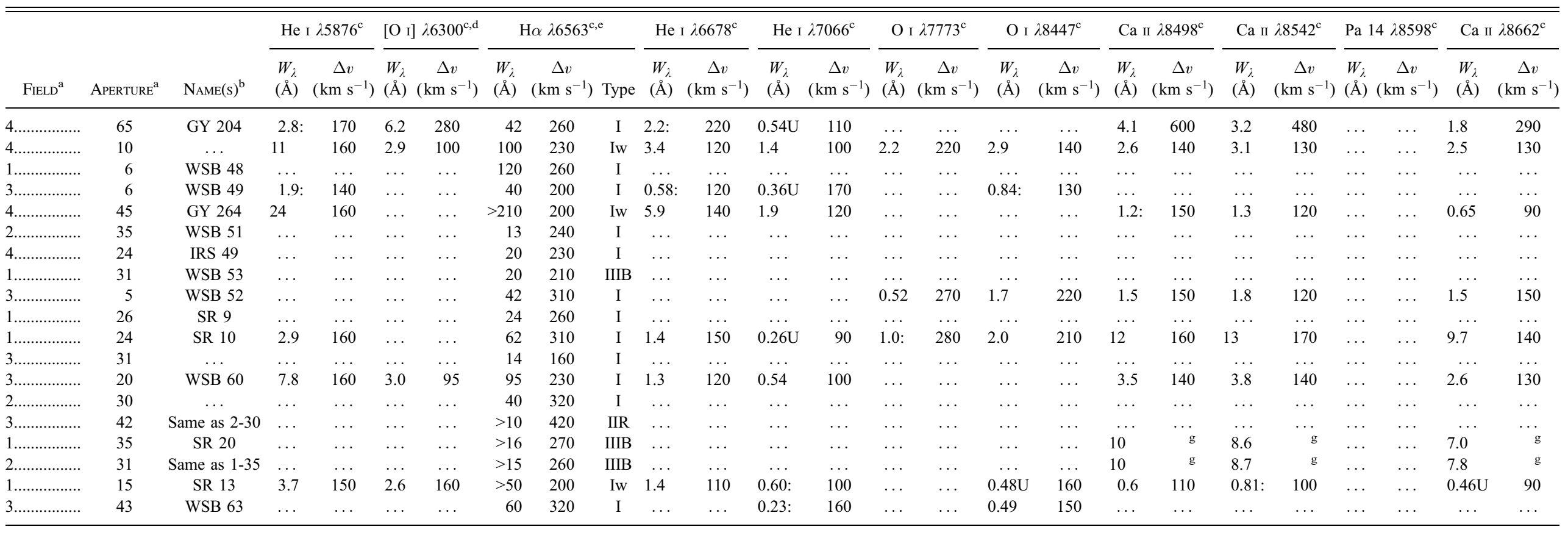

Noте.-Table 3 is also available in machine-readable form in the electronic edition of the Astronomical Journal.

${ }^{a}$ Hydra field and aperture number of observation.

Source names as noted in Table 2.

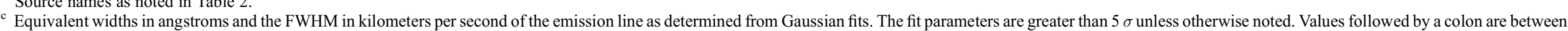
3 and $5 \sigma$, and values followed by a "U" are upper limits. The values for FWHM have not been deconvolved by the instrumental response.

${ }^{\mathrm{d}}$ The $[\mathrm{O} \mathrm{I}]$ line at $6363 \AA$ is also observed in emission but is much weaker.

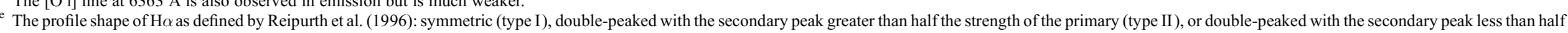

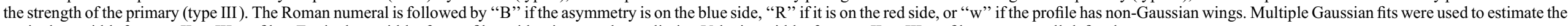
equivalent width for type II or III profiles. Equivalent widths for profiles with wings are lower limits. Velocity widths for type II or III profiles are not well defined.

${ }^{1} \mathrm{~Pa} 19$ at $8413 \AA$ was also detected in the spectrum of WL 18 with $W \lambda=1.9: \AA$ and $\Delta v=180: \mathrm{km} \mathrm{s}^{-1}$

g The Ca II emission lines display double-peaked type IIIB profiles similar to $\mathrm{H} \alpha$. 

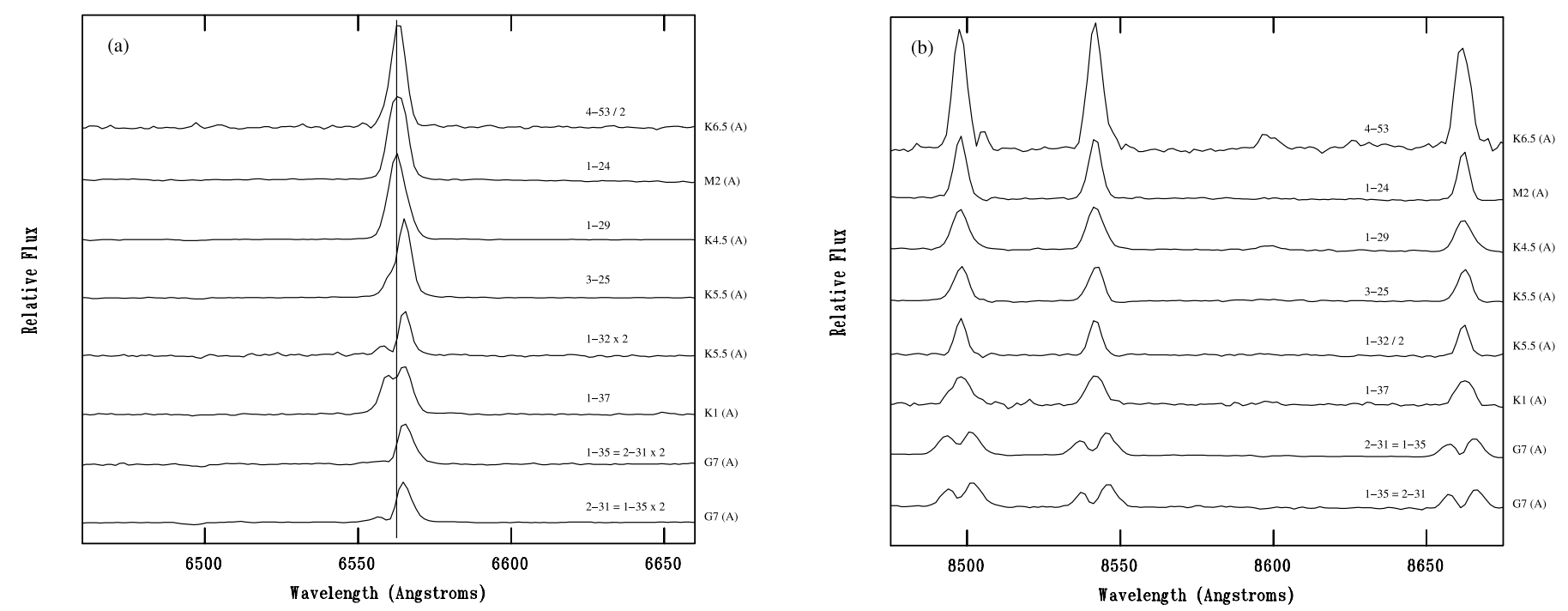

FIG. 5.-Expanded view of $\mathrm{H} \alpha$ and $\mathrm{Ca}$ II emission lines in seven CTTSs labeled with their field and aperture numbers (Table 2) and spectral types. The bottom two spectra in each panel are from the same source observed on two different nights. (a) Structure of the $\mathrm{H} \alpha$ emission line, with the rest wavelength of $\mathrm{H} \alpha$ marked as a reference. (b) Ca II triplet for the same objects.

the core. Only 14 members are seen in projection outside the lowest contour, corresponding to $N_{\mathrm{LTE}}(13)=6 \times 10^{14} \mathrm{~cm}^{-2}$.

\subsection{Hertzsprung-Russell Diagram}

With the benefit of the spectroscopic data, we can investigate the ages and masses of the association members identified by this study. The intrinsic colors, bolometric corrections, and temperature scale for dwarf stars were derived from the works of Schmidt-Kaler (1982) for B8-K5 stars and from Bessell (1991) for K5-M7 stars. We note that the assumption of dwarf, rather than subgiant, surface gravities will overestimate $T_{\text {eff }}$ for stars with spectral types of $\mathrm{G} 5-\mathrm{K} 5$ by $<250 \mathrm{~K}$ and underestimate $T_{\text {eff }}$ for stars with spectral types of M2-M 8 by $<200 \mathrm{~K}$. For the B3 star Source 1, an effective temperature of $T_{\text {eff }}=19,000$ and an $L_{*}=1500 L_{\odot}$ was adopted (e.g., Lada \& Wilking 1984). For the M8 brown dwarf candidates, we assumed values of $T_{\text {eff }}=2500,(R-I)_{0}=2.5$, and $\mathrm{BC}(I)=-1.7$ that are consistent with recent studies of very low mass stars (Dahn et al. 2002; Hawley et al. 2002). Bolometric luminosities were computed from the $I$-band magnitudes in the following way. Magnitudes were dereddened using the $(R-I)$ color excess derived from the observed minus intrinsic $(R-I)$ color for the corresponding spectral type and using the reddening law $A_{v} / E(R-I)=6.25$ (Cohen et al. 1981). In a few cases the errors in the photometry and/or spectral classification yielded slightly negative values for the extinction, and an extinction of 0.0 was assumed. Absolute $I$ magnitudes were then computed, assuming a distance of $150 \mathrm{pc}$ (de Zeeuw et al. 1999), and converted to bolometric magnitudes using the appropriate bolometric correction for the observed spectral type. The stellar luminosity was computed using $\log \left(L / L_{\odot}\right)=$ $1.89-0.4 M_{\mathrm{bol}}$, assuming $M_{\mathrm{bol}, \odot}=4.74$ (Livingston 1999). Due to the dominance of emission lines in the spectrum of WL 18, we adopted the luminosity estimated by Bontemps et al. (2001) from the $J$-band flux and $(J-H)$ color, adjusted to a distance of $150 \mathrm{pc}$.

The resulting H-R diagram is presented in Figure 7 for 84 association members relative to the theoretical tracks and isochrones of D'Antona \& Mazzitelli (1997) and F. D'Antona \& I. Mazzitelli (1998, private communication). Excluded from the diagram is the B3 star Source 1, the A7 star VSSG 14, and objects GY 59 and GY 326, which lack $R$-band photometry and therefore estimates for $A_{v}$ and $\log L$. CTTSs are indicated by filled diamonds; open diamonds represent association members with weak or no $\mathrm{H} \alpha$ emission that would correspond to WTTSs or post-T Tauri stars. The errors in $T_{\text {eff }}$ are estimated to be $\pm 105 \mathrm{~K}$ for $\mathrm{K}-\mathrm{M}$ stars with equal contributions from the uncertainties in the spectral classification and systematic offsets in the temperature scale. The uncertainty in $\log L$ is estimated to be 0.12 dex, assuming uncertainties in the $R$ and $I$ photometry of $0.04 \mathrm{mag}$, in the distance modulus of $0.15 \mathrm{mag}$, and in the bolometric correction of $0.1 \mathrm{mag}$. The masses and ages interpolated from the models are given in Table 4 with uncertainties due to random errors of $20 \%$ in mass and 0.25 dex in log (age). Comparisons with the Baraffe et al. (1998) models suggest systematic errors between different sets of models of $\sim 50 \%$ in mass and $\sim 0.5$ dex in $\log$ (age) (e.g., Hillenbrand \& White 2004).

The median age of the association members in Figure 7 is 2.1 Myr. There is no significant difference observed in the median age of the CTTS and non-CTTS association members. Following the analysis of Preibisch \& Zinnecker (1999), the apparent age spread observed in Figure 7 is consistent with all the association members having roughly the same age (1-3 Myr) with the scatter in the age accounted for by the errors noted above and unresolved binaries.

\section{IMPLICATIONS FOR THE STAR FORMATION HISTORY}

The association members identified in this study are premain-sequence objects distributed over a $1.3 \mathrm{deg}^{2}$ area $\left(6.8 \mathrm{pc}^{2}\right)$ and lie within a projected radius of $2 \mathrm{pc}$ of the highest extinction regions of the main $\rho$ Oph cloud (see Fig. 6). The fact that our derived visual extinctions are generally low ( $80 \%$ of the association members have $A_{v} \leq 5 \mathrm{mag}$ ) indicates that the majority of our sample lies at the surface of the cloud. Recent spectroscopic studies of YSOs in the $\rho$ Oph cloud have focused on the embedded population in the $1 \mathrm{pc} \times 2 \mathrm{pc}$ centrally condensed core and have necessarily been conducted at near-infrared wavelengths (Greene \& Meyer 1995; Luhman \& Rieke 1999; WGM99; Natta et al. 2002). These studies have consistently derived ages between 0.1 and $1 \mathrm{Myr}$ when using the D'Antona \& Mazzitelli (1997) tracks and isochrones, with a median age of 0.3 Myr. After considering our uncertainties, we conclude that the distributed 
TABLE 4

Association Members

\begin{tabular}{|c|c|c|c|c|c|c|c|c|c|}
\hline Field & Aperture & Name & $\begin{array}{c}A_{v} \\
(\mathrm{mag})\end{array}$ & $\begin{array}{l}M(I) \\
(\mathrm{mag})\end{array}$ & $\begin{array}{c}\log T_{\text {eff }} \\
(\mathrm{K})\end{array}$ & $\log \left(L / L_{\odot}\right)$ & $\begin{array}{c}M_{*}^{\mathrm{a}} \\
\left(M_{\odot}\right)\end{array}$ & $\begin{array}{c}\log (\text { age })^{a} \\
(\text { Myr })\end{array}$ & Notes $^{\mathrm{b}}$ \\
\hline 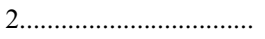 & 15 & & 2.6 & 6.26 & 3.512 & -0.76 & 0.19 & 6.09 & li, ext \\
\hline 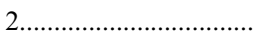 & 14 & & 2.1 & 8.03 & 3.525 & -1.50 & 0.27 & 7.28 & ext \\
\hline 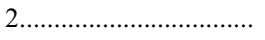 & 10 & & 0.8 & 8.13 & 3.477 & -1.38 & 0.14 & 6.57 & ha \\
\hline $1 \ldots \ldots \ldots \ldots \ldots \ldots \ldots \ldots$ & 7 & SR 22 & 0.0 & 7.00 & 3.488 & -0.98 & 0.15 & 6.18 & ha, $x$ \\
\hline 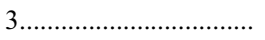 & 15 & WLY 2-3 & 4.9 & 6.04 & 3.544 & -0.74 & 0.31 & 6.33 & ha, $x$, ext \\
\hline (.......................... & 32 & & 2.6 & 6.79 & 3.512 & -0.98 & 0.20 & 6.32 & $x$, li, ext \\
\hline 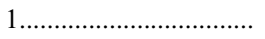 & 22 & VSS 23 & 5.1 & 3.43 & 3.630 & 0.25 & 0.53 & 5.59 & $\mathrm{x}$, ext \\
\hline $2 \ldots \ldots \ldots \ldots \ldots \ldots \ldots \ldots$ & 25 & WLY 2-11 & 2.3 & 7.25 & 3.477 & -1.03 & 0.14 & 6.17 & ha, ext, $x$ \\
\hline 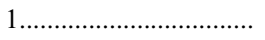 & 29 & SR 4 & 0.5 & 5.15 & 3.650 & -0.43 & 0.91 & 7.01 & ha, x, li \\
\hline $1 \ldots \ldots \ldots \ldots+\ldots \ldots \ldots+\ldots$ & 38 & GSS 20 & 6.0 & 3.54 & 3.630 & 0.21 & 0.54 & 5.64 & $\mathrm{x}$, ext \\
\hline $3 \ldots \ldots \ldots \ldots \ldots \ldots \ldots \ldots$ & 14 & & 1.0 & 7.36 & 3.488 & -1.12 & 0.16 & 6.33 & li \\
\hline 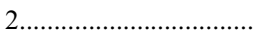 & 1 & Chini 8 & 0.0 & 7.12 & 3.462 & -0.93 & 0.12 & 5.57 & ha, $x$, li \\
\hline 4 & 28 & & 5.3 & 5.93 & 3.525 & -0.66 & 0.22 & 6.06 & $\mathrm{x}$, ext \\
\hline $1 \ldots \ldots \ldots \ldots \ldots \ldots \ldots \ldots .$. & 13 & VSSG 19 & 3.4 & 5.05 & 3.544 & -0.35 & 0.27 & 5.83 & $x$, ext \\
\hline 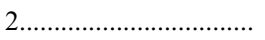 & 19 & & 2.1 & 6.63 & 3.488 & -0.83 & 0.15 & 6.01 & ha, li, ext \\
\hline 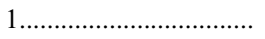 & 5 & GSS 28 & 2.8 & 4.28 & 3.650 & -0.08 & 0.79 & 6.31 & ha, $x$, li, ext \\
\hline 4 & 39 & & 1.9 & 6.06 & 3.512 & -0.68 & 0.18 & 6.01 & li, ext \\
\hline $2 \ldots \ldots \ldots \ldots \ldots \ldots \ldots \ldots$ & 11 & & 0.1 & 7.39 & 3.482 & -1.11 & 0.15 & 6.30 & $\mathrm{x}, \mathrm{li}$ \\
\hline 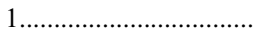 & 25 & WSB 28 & 2.9 & 5.48 & 3.544 & -0.52 & 0.29 & 6.02 & ha, $x$, ext \\
\hline 4 & 16 & GY 5 & 2.8 & 8.27 & 3.462 & -1.39 & 0.11 & 6.47 & ha, $x$, ext \\
\hline 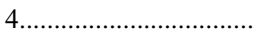 & 41 & GY 3 & 0.0 . & 9.74 & 3.398 & -1.33 & $<0.04$ & $<5.0$ & ha, $x$ \\
\hline 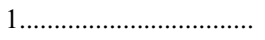 & 30 & GSS 31 & 6.0 & 2.26 & 3.752 & 0.82 & 2.10 & 6.50 & $\mathrm{x}$, ext \\
\hline 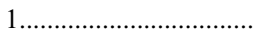 & 28 & DoAr 25 & 2.9 & 3.97 & 3.638 & 0.03 & 0.64 & 5.97 & ha, $x$, li, ext \\
\hline $2 \ldots \ldots \ldots \ldots \ldots \ldots \ldots \ldots$ & 27 & EL 24 & 7.5 & 3.57 & 3.630 & 0.19 & 0.54 & 5.66 & ha, $x$, ext \\
\hline 4 & 18 & & 2.2 & 8.64 & 3.488 & -1.64 & 0.16 & 7.00 & ext \\
\hline 4 & 25 & & 1.3 & 9.12 & 3.462 & -1.73 & 0.09 & 6.74 & $\mathrm{x}$ \\
\hline 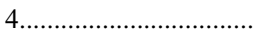 & 66 & GY 37 & 3.4 & 9.95 & 3.477 & -2.11 & 0.10 & 7.31 & ha, $x$, ext \\
\hline 4 & 27 & GY 59 & $\ldots$ & $\ldots$ & 3.491 & $\ldots$ & $\ldots$ & $\ldots$ & $\mathrm{x}$ \\
\hline 2 & 36 & WSB 34 & 0.8 & 7.80 & 3.488 & -1.30 & 0.16 & 6.55 & ha \\
\hline $1 \ldots \ldots \ldots \ldots \ldots \ldots \ldots \ldots$ & 2 & Source 1 & 13 & -1.15 & 4.278 & 3.20 & 5.00 & 7.60 & $x$, ext \\
\hline $4 \ldots \ldots \ldots \ldots \ldots \ldots \ldots \ldots \ldots$ & 4 & & 1.9 & 8.51 & 3.477 & -1.53 & 0.14 & 6.74 & ha, $x$, ext \\
\hline $2 \ldots \ldots \ldots \ldots \ldots \ldots \ldots \ldots$ & 29 & WSB 37 & 0.8 & 7.00 & 3.477 & -0.93 & 0.14 & 6.04 & ha, $x$, li \\
\hline 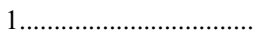 & 21 & & 4.3 & 4.83 & 3.591 & -0.29 & 0.48 & 6.08 & ext, $x$ \\
\hline 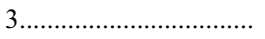 & 22 & & 0.4 & 7.74 & 3.488 & -1.28 & 0.16 & 6.52 & li, $x$ \\
\hline 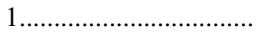 & 20 & GY 112 & 2.4 & 5.92 & 3.525 & -0.65 & 0.22 & 6.05 & $\mathrm{x}$, ext \\
\hline 4 & 13 & & 4.6 & 6.87 & 3.525 & -1.03 & 0.24 & 6.52 & li, ext \\
\hline $1 \ldots \ldots \ldots \ldots \ldots \ldots \ldots \ldots \ldots$ & 36 & WSB 38 & 7.7 & 1.87 & 3.765 & 0.98 & 2.20 & 6.45 & ha, $x$, li, ext \\
\hline 4 & 30 & & 2.8 & 7.62 & 3.488 & -1.23 & 0.16 & 6.46 & $\mathrm{x}, \mathrm{li}$, ext \\
\hline 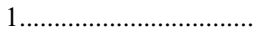 & 32 & WSB 40 & 5.6 & 3.68 & 3.630 & 0.15 & 0.55 & 5.71 & ha, ext \\
\hline 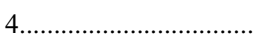 & 53 & WL 18 & $10.4^{\mathrm{c}}$ & 6.89 & 3.612 & $-0.66^{\mathrm{c}}$ & 0.78 & 7.10 & ha, $x$, ext \\
\hline 2 & 38 & & 1.7 & 7.89 & 3.488 & -1.34 & 0.16 & 6.60 & ext \\
\hline 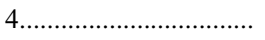 & 46 & & 8.8 & 5.16 & 3.602 & -0.42 & 0.61 & 6.45 & ha, ext, $x$ \\
\hline 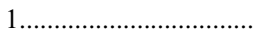 & 37 & SR 24s & 5.5 & 3.68 & 3.705 & 0.21 & 1.40 & 6.64 & ha, $x$, ext \\
\hline 4 & 63 & & 2.6 & 7.89 & 3.488 & -1.33 & 0.16 & 6.59 & ext \\
\hline $4 \ldots \ldots \ldots \ldots \ldots \ldots \ldots \ldots$ & 65 & GY 204 & 0.5 & 9.25 & 3.462 & -1.78 & 0.09 & 6.77 & ha, $x$ \\
\hline 4 & 37 & & 6.7 & 6.27 & 3.535 & -0.82 & 0.27 & 6.34 & ext \\
\hline 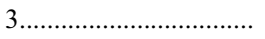 & 44 & WSB 45 & 0.0 & 7.43 & 3.488 & -1.15 & 0.16 & 6.37 & li, $x$ \\
\hline $1 \ldots \ldots \ldots \ldots \ldots \ldots \ldots \ldots$ & 9 & WSB 46 & 0.6 & 6.01 & 3.544 & -0.73 & 0.31 & 6.31 & $\mathrm{x}$ \\
\hline 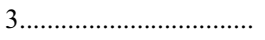 & 17 & & 8.6 & 4.00 & 3.705 & 0.08 & 1.30 & 6.81 & ext \\
\hline 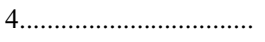 & 10 & & 4.4 & 6.97 & 3.512 & -1.04 & 0.20 & 6.40 & ha, ext \\
\hline $1 \ldots \ldots \ldots \ldots \ldots \ldots \ldots \ldots$ & 6 & WSB 48 & 0.0 & 7.35 & 3.491 & -1.18 & 0.16 & 6.42 & ha \\
\hline$\ldots$ & $\ldots$ & SR 12 & 2.4 & 3.76 & 3.580 & 0.15 & 0.31 & 4.99 & $\mathrm{x}$, ext \\
\hline 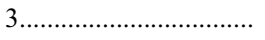 & 6 & WSB 49 & 0.3 & 7.24 & 3.493 & -1.10 & 0.16 & 6.34 & ha, $x$, li \\
\hline 4 & 45 & GY 264 & 0.0 & 9.84 & 3.398 & -1.37 & $<0.04$ & $<5.0$ & ha, $x$ \\
\hline 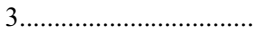 & 7 & & 0.0 & 8.44 & 3.482 & -1.53 & 0.15 & 6.80 & $\mathrm{x}$ \\
\hline $2 \ldots \ldots \ldots \ldots \ldots \ldots \ldots \ldots \ldots \ldots \ldots \ldots \ldots \ldots \ldots \ldots \ldots$ & 20 & & 1.1 & 7.75 & 3.544 & -1.43 & 0.36 & 7.46 & $\mathrm{x}$ \\
\hline 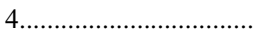 & 22 & GY 284 & 4.8 & 7.45 & 3.519 & -1.25 & 0.24 & 6.80 & $\mathrm{x}$, ext \\
\hline $2 \ldots \ldots \ldots \ldots \ldots \ldots \ldots \ldots$ & 6 & WSB 50 & 1.9 & 7.04 & 3.488 & -1.00 & 0.15 & 6.20 & $x$, li, ext \\
\hline 4 & 54 & & 3.0 & 6.05 & 3.535 & -0.73 & 0.26 & 6.22 & li, ext \\
\hline $2 \ldots \ldots \ldots \ldots \ldots \ldots \ldots \ldots$ & 35 & WSB 51 & 4.3 & 3.85 & 3.621 & 0.08 & 0.51 & 5.74 & ha, $x$, li, ext \\
\hline 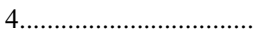 & 24 & WLY 2-49 & 9.6 & 3.88 & 3.630 & 0.07 & 0.57 & 5.82 & ha, $x$, ext \\
\hline 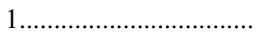 & 31 & WSB 53 & 3.7 & 3.94 & 3.630 & 0.04 & 0.58 & 5.87 & ha, ext \\
\hline 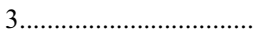 & 5 & WSB 52 & 7.1 & 3.90 & 3.638 & 0.06 & 0.63 & 5.92 & ha, $x$, li, ext \\
\hline 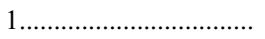 & 26 & SR 9 & 0.0 & 5.38 & 3.638 & -0.53 & 0.85 & 7.10 & ha, $x$, li \\
\hline $2 \ldots \ldots \ldots \ldots \ldots \ldots \ldots \ldots \ldots \ldots \ldots \ldots \ldots \ldots \ldots \ldots \ldots$ & 34 & & 4.1 & 5.04 & 3.544 & -0.34 & 0.27 & 5.82 & $\mathrm{x}$, ext \\
\hline 4 & 64 & GY 326 & $\ldots$ & $\ldots$ & 3.544 & $\ldots$ & $\ldots$ & $\ldots$ & $\mathrm{x}$ \\
\hline$\ldots \ldots \ldots \ldots \ldots$ & 21 & & 0.4 & 7.99 & 3.488 & -1.37 & 0.16 & 6.64 & li \\
\hline
\end{tabular}


TABLE 4-Continued

\begin{tabular}{|c|c|c|c|c|c|c|c|c|c|}
\hline Field & Aperture & Name & $\begin{array}{c}A_{v} \\
(\mathrm{mag})\end{array}$ & $\begin{array}{l}M(I) \\
\text { (mag) }\end{array}$ & $\begin{array}{c}\log T_{\text {eff }} \\
(\mathrm{K})\end{array}$ & $\log \left(L / L_{\odot}\right)$ & $\begin{array}{l}M_{*}^{\mathrm{a}} \\
\left(M_{\odot}\right)\end{array}$ & $\begin{array}{c}\log (\text { age })^{\mathrm{a}} \\
(\mathrm{Myr})\end{array}$ & Notes $^{\mathrm{b}}$ \\
\hline 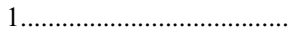 & 14 & VSSG 14 & 6.7 & 2.24 & 3.895 & 0.94 & 1.70 & 7.15 & $\mathrm{x}$, ext \\
\hline 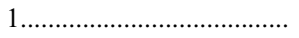 & 10 & ROX 31 & 5.0 & 3.79 & 3.597 & 0.13 & 0.38 & 5.53 & $\mathrm{x}$, ext \\
\hline ..................... & 24 & SR 10 & 0.1 & 5.99 & 3.544 & -0.73 & 0.31 & 6.31 & ha, $x$ \\
\hline (n)................... & 34 & WSB 58 & 0.9 & 7.00 & 3.482 & -0.95 & 0.14 & 6.11 & $\mathrm{x}$ \\
\hline 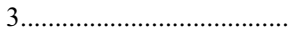 & 31 & & 0.9 & 7.11 & 3.477 & -0.97 & 0.14 & 6.09 & ha, x, li \\
\hline ....... & 3 & & 3.0 & 6.21 & 3.525 & -0.77 & 0.23 & 6.18 & li, ext \\
\hline 2 & 21 & & 3.5 & 6.27 & 3.491 & -0.75 & 0.15 & 5.77 & ext \\
\hline 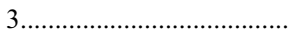 & 11 & ROX 32 & 3.5 & 5.69 & 3.525 & -0.56 & 0.21 & 5.94 & $x$, li, ext \\
\hline З & 20 & WSB 60 & 2.0 & 7.38 & 3.488 & -1.13 & 0.16 & 6.34 & ha, ext \\
\hline 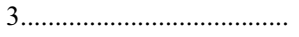 & 12 & & 1.9 & 6.53 & 3.525 & -0.90 & 0.24 & 6.34 & ext \\
\hline 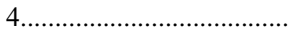 & 3 & & 4.0 & 7.27 & 3.512 & -1.17 & 0.21 & 6.59 & ext \\
\hline 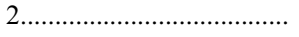 & 37 & & 4.7 & 6.24 & 3.535 & -0.81 & 0.27 & 6.32 & ext, $x$ \\
\hline (n).................... & 30 & & 6.8 & 4.62 & 3.638 & -0.22 & 0.76 & 6.44 & ha, ext, $x$ \\
\hline …............... & 4 & & 4.8 & 5.87 & 3.544 & -0.68 & 0.30 & 6.24 & ext, $x$ \\
\hline 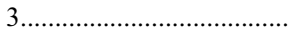 & 39 & & 0.5 & 8.61 & 3.477 & -1.57 & 0.14 & 6.79 & li \\
\hline . & 57 & & 5.3 & 6.88 & 3.512 & -1.01 & 0.20 & 6.36 & ext \\
\hline 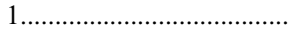 & 35 & SR 20 & 5.7 & 2.08 & 3.747 & 0.89 & 2.20 & 6.41 & ha, $x$, ext \\
\hline (n) & 23 & & 1.4 & 7.73 & 3.482 & -1.25 & 0.15 & 6.45 & li, $x$ \\
\hline 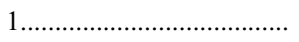 & 15 & SR 13 & 0.0 & 5.07 & 3.491 & -0.27 & 0.13 & 4.55 & ha, $x$ \\
\hline 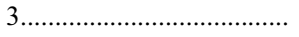 & 43 & WSB 63 & 3.8 & 5.37 & 3.553 & -0.48 & 0.34 & 6.04 & ha, li, ext, $x$ \\
\hline 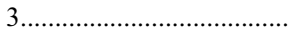 & 37 & & 0.0 & 8.61 & 3.462 & -1.52 & 0.10 & 6.57 & li \\
\hline
\end{tabular}

NoтE.-Table 4 is also available in machine-readable form in the electronic edition of the Astronomical Journal.

${ }^{a}$ Masses and ages derived relative to the theoretical tracks and isochrones of D’Antona \& Mazzitelli (1997) and F. D'Antona \& I. Mazzitelli (1998, private communication).

${ }^{\mathrm{b}}$ Association membership established through the presence of $\mathrm{H} \alpha$ emission (ha), X-ray emission (x), lithium absorption (li), or location above the main sequence and $A_{v}>1.5 \mathrm{mag}$ (ext).

${ }^{c}$ Due to strong emission lines in the $R$ and $I$ bands relative to the continuum, the $A_{v}$ and luminosity estimates are taken from Bontemps et al. (2001) using the $(J-H)$ color and adjusted to a distance of $150 \mathrm{pc}$.

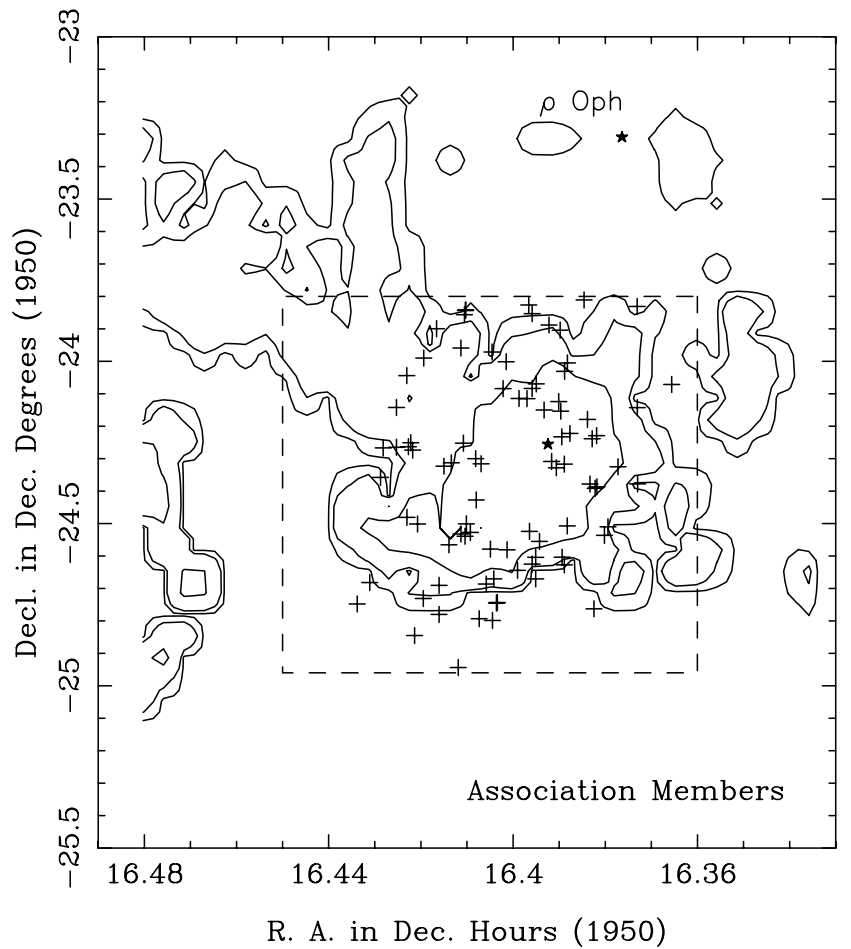

FIG. 6.-Distribution of association members relative to contours of ${ }^{13} \mathrm{CO}$ column density. The contours were computed from Loren (1989) assuming LTE and $T_{\mathrm{ex}}=25 \mathrm{~K}$. The values of the contours in units of $\mathrm{cm}^{-2}$ are $6 \times 10^{14}$, $3 \times 10^{15}$, and $1.5 \times 10^{16}$; the lowest contour delineates the outer boundary of the dark cloud. The dashed box outlines the field included in our Hydra observations. Star symbols mark the locations of the star $\rho$ Oph A (labeled) and the embedded association member Oph S1 in the L1688 core. population is significantly older than that in the more highly extincted cloud core.

The question then arises as to the origin of this more distributed population. One explanation is that these objects formed in the dense core and have diffused or been ejected to the surface of the cloud. However, the space motion of a YSO that acquires the velocity dispersion of the molecular gas in which it formed $\left(1-2 \mathrm{~km} \mathrm{~s}^{-1}\right)$ would not be high enough to move an object to the projected distances from the core of $>1 \mathrm{pc}$ that we observe for some association members. Alternatively, the ejection of very low mass stars and brown dwarfs from small groups has been proposed for the origin of widely distributed WTTSs and of substellar objects (Sterzik \& Durisen 1995; Reipurth \& Clarke 2001; Bate et al. 2002). In this case, one would predict that the lowest mass association members in our sample would display a higher velocity dispersion (and thus a broader spatial distribution) than YSOs in the core. We have analyzed the distribution of the higher mass association members $\left(M>0.5 M_{\odot}\right)$ relative to the lower mass members $\left(M<0.5 M_{\odot}\right)$ using a twodimensional Kolmogorov-Smirnov (K-S) test. The K-S statistic is consistent with the two populations being drawn from the same parent population. Our data do not lend support to the ejection hypothesis; however, our sample includes only eight brown dwarf candidates. ${ }^{4}$

A second possibility is that the association members at the cloud edges are near their birth sites and that the dense gas in L1688 was once distributed over a larger area than we see today. It has been proposed that star formation in Upper Sco was triggered about 5 Myr ago by the passage of an expanding $\mathrm{H}$ I

\footnotetext{
${ }^{4}$ Radial velocity measurements are required for a detailed analysis of the kinematics of these stars to reject the hypothesis that they were ejected from the cloud as the result of the dissolution of small stellar groups.
} 


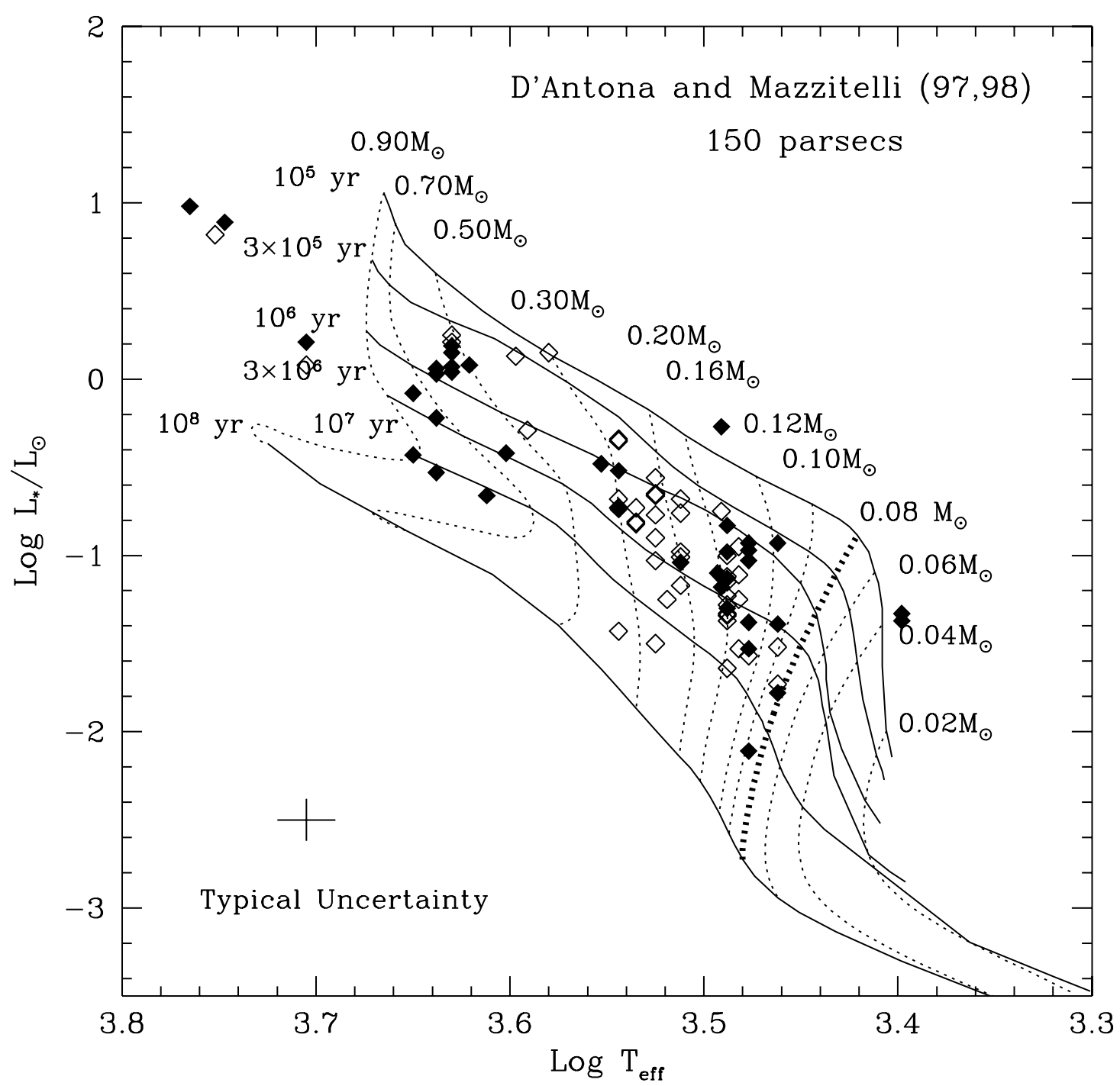

FIG. 7.-H-R diagram for the $\rho$ Oph association members with optically determined spectral types. The filled diamonds indicate the positions of CTTSs $[\mathrm{EW}(\mathrm{H} \alpha)>10 \AA]$, and the open diamonds correspond to WTTSs or post-T Tauri stars relative to the theoretical tracks of D'Antona \& Mazzitelli (1997) and F. D'Antona \& I. Mazzitelli (1998, private communication). Isochrones shown as solid lines are $10^{5}, 3 \times 10^{5}, 10^{6}, 3 \times 10^{6}$, $10^{7}$, and $10^{8}$ yr. Evolutionary tracks from 0.02 to $0.90 M_{\odot}$ are shown by dashed lines. The bold dashed line marks the evolutionary track for a star at the hydrogen-burning limit. The typical error bar for a candidate is shown at the bottom left and is $\pm 0.015 \mathrm{dex}$ in $\log T_{\mathrm{eff}}$ and $\pm 0.12 \mathrm{dex}$ in $\log \left(L_{\mathrm{bol}} / L_{\odot}\right)$.

shell driven by massive stars in the Upper Centaurus-Lupus association (de Geus 1992). This age is consistent with that of high-mass stars in Upper Sco derived from a color-magnitude diagram and from the main-sequence turnoff (Preibisch et al. 2002; see models by Bertelli et al. 1994). The implied age of the low-mass stars in Upper Sco is also $5 \mathrm{Myr}$, but only after corrections are made for unresolved binaries (Preibisch \& Zinnecker 1999). A comparison of Figure 7 with H-R diagrams for low-mass members of the Upper Scorpius subgroup, using the same set of theoretical evolutionary tracks and isochrones and not corrected for unresolved binaries, shows no discernible difference in age or age spread (e.g., Preibisch \& Zinnecker 1999; Preibisch et al. 2001, 2002). This is not too surprising given that the $\rho \mathrm{Oph}$ cloud is ringed by high-mass members of Upper Sco such as the multiple star $\rho$ Oph and $\sigma$ Sco to the west and $22 \mathrm{Sco}, \alpha \operatorname{Sco}$, and $\tau$ Sco to the east. Therefore, one could regard the distributed population we observe as either low-mass members of the Upper Scorpius subgroup or older members of the $\rho$ Oph cloud whose formation was contemporaneous with stars in the Upper Scorpius subgroup. In either scenario, ultra- violet radiation and the passage of an expanding shell from massive stars in the Upper Scorpius subgroup within the last 2.5 Myr would have stripped away the outer skin of the $\rho$ Oph cloud, revealing the present population and perhaps triggering a second episode of star formation in the centrally condensed core (de Geus 1992).

\section{SUMMARY}

We have analyzed optical spectra for 139 candidate CTTSs that extend over a $1.3 \mathrm{deg}^{2}$ area in the direction of the main $\rho$ Oph cloud. Of the 131 stars for which spectral types and surface gravities are estimated, 6 are identified as giants and 79 are classified as M stars. Association with the cloud is established for 88 objects by the presence of optical emission lines or lithium absorption in the spectra, by location in the H-R diagram, and/or by previously reported X-ray emission. Thirty-nine of the stars display strong $\mathrm{H} \alpha$ emission ( $\mathrm{EW}>10 \AA$ ) characteristic of CTTSs. These emission lines are broad (FWHM $\sim 250 \mathrm{~km} \mathrm{~s}^{-1}$ ), consistent with their origin in magnetospheric accretion columns. In addition, asymmetries in the $\mathrm{H} \alpha$ profiles and the presence of 
forbidden lines observed in 16 objects are a signpost of mass outflows. A subset of the CTTSs also displays emission from the Ca II triplet that is strongest in the most massive CTTSs and, in one instance, self-absorbed. Two of the strongest emission-line objects are young, X-ray-emitting brown dwarf candidates with M8 spectral types.

Association members are distributed in the lower extinction regions surrounding the high-extinction core. An H-R diagram suggests a median age of 2.1 Myr with an apparent age spread due mainly to uncertainties in the calculated luminosity and the presence of unresolved binaries. This age is significantly older than the median age of $0.3 \mathrm{Myr}$ for objects in the high-extinction core. There is no difference in median age between the CTTS and non-CTTS association members, and there are no statistically significant correlations between the age or mass of an association member and its projected distance from the core center. The age and distribution of this population is indistinguishable from that of low-mass stars in the Upper Scorpius subgroup. We propose that these stars formed in what was once a larger $\rho$ Oph cloud. In this instance, star formation in the outer regions of the $\rho$ Oph cloud would have been triggered by the same event that initiated star formation in Upper Sco.

We thank Di Harmer for obtaining the Hydra data through the WIYN Queue Observing Program and for assistance with the data reduction. Lori Allen provided helpful insight into the spectraltyping process and made available the bulk of the spectral standards used in this study in digital form. We gratefully acknowledge Eric Mamajek for helpful discussions. M. R. M. acknowledges support for this work through a Cottrell Scholar's Award from the Research Corporation. B. W. and J. R. acknowledge support from NSF AST 98-20898 to the University of Missouri-St. Louis and from the Missouri Research Board.
Alcalá, J. M., Covino, E., Torres, G., Sterzik, M. F., Pfeiffer, M. J., \& Neuhäuser R. 2000, A\&A, 353, 186

Alcalá, J. M., et al. 1996, A\&AS, 119, 7

Allen, L. E. 1995, Ph.D. thesis, Univ. Massachusetts

Allen, L. E., \& Strom, K. M. 1995, AJ, 109, 1379

André, P., \& Montmerle, T. 1994, ApJ, 420, 837

André, P., Montmerle, T., \& Feigelson, E. D. 1987, AJ, 93, 1182

Baraffe, I., Charbrier, G., Allard, F., \& Hauschuldt, P. H. 1998, A\&A, 337, 403

Bate, M. R., Bonnell, I. A., \& Bromm, V. 2002, MNRAS, 332, L65

Bertelli, G., Bressan, A., Chiosi, C., Fagotto, F., \& Nasi, E. 1994, A\&AS, 106, 275

Bessell, M. S. 1991, AJ, 101, 662

Blaauw, A. 1991, in The Physics of Star Formation and Early Stellar Evolution, ed. C. J. Lada \& N. D. Kylafis (NATO ASI Ser. C, 342; Dordrecht: Kluwer), 125

Bontemps, S., et al. 2001, A\&A, 372, 173

Bouvier, J., \& Appenzeller, I. 1992, A\&AS, 92, 48

Casanova, S., Montmerle, T., Feigelson, E. D., \& André, P. 1995, ApJ, 439, 752

Chini, R. 1981, A\&A, 99, 346

Cohen, J. G., Frogel, J. A., Persson, S. E., \& Elias, J. H. 1981, ApJ, 249, 481

Cohen, M., \& Kuhi, L. V. 1979, ApJS, 41, 743

Cutri, R. M., et al. 2003, 2MASS All-Sky Catalog of Point Sources (Pasadena: IPAC)

Dahn, C., et al. 2002, AJ, 124, 1170

D'Antona, F., \& Mazzitelli, I. 1997, Mem. Soc. Astron. Italiana, 68, 807

de Geus, E. J. 1992, A\&A, 262, 258

de Zeeuw, P. T., Hoogerwerf, R., de Bruijne, J. H. J., Brown, A. G. A., \& Blaauw, A. 1999, AJ, 117, 354

Dolidze, M. V., \& Arakelyan, M. A. 1959, Soviet Astron., 3, 434

Edwards, S., Cabrit, S., Strom, S., Heyer, I., Strom, K., \& Anderson, E. 1987, ApJ, 321, 473

Elias, J. H. 1978, ApJ, 224, 453

Feigelson, E. D. 1996, ApJ, 468, 306

Festin, L. 1998, A\&A, 336, 883

Gagné, M., Skinner, S. L., \& Daniel, K. J. 2004, ApJ, 613, 393

Grasdalen, G. L., Strom, K. M., \& Strom, S. E. 1973, ApJ, 184, L53

Greene, T. P., \& Meyer, M. R. 1995, ApJ, 450, 233

Greene, T. P., \& Young, E. T. 1992, ApJ, 395, 516

Grosso, N., Montmerle, T., Bontemps, S., André, P., \& Feigelson, E. D. 2000, A\&A, 359, 113

Hartigan, P. 1993, AJ, 105, 1511

Hartmann, L., Hewett, R., \& Calvet, N. 1994, ApJ, 426, 669

Hawley, S., et al. 2002, AJ, 123, 3409
Hillenbrand, L. A. 1997, AJ, 113, 1733

Hillenbrand, L. A., \& White, R. J. 2004, ApJ, 604, 741

Imanishi, K., Koyama, K., \& Tsuboi, Y. 2001, ApJ, 557, 747

Kirkpatrick, J. D., Henry, T. J., \& McCarthy, D. W. 1991, ApJS, 77, 417

Lada, C. J., \& Wilking, B. A. 1984, ApJ, 287, 610

Landolt, A. U. 1992, AJ, 104, 340

Leous, J. A., Feigelson, E. D., André, P., \& Montmerle, T. 1991, ApJ, 379, 683

Livingston, W. C. 1999, in Allen's Astrophysical Quantities, ed. A. Cox (4th ed.; New York: AIP), 341

Loren, R. B. 1989, ApJ, 338, 925

Luhman, K. L. 1999, ApJ, 525, 466

Luhman, K. L., Liebert, J., \& Rieke, G. H. 1997, ApJ, 489, L165

Luhman, K. L., \& Rieke, G. H. 1999, ApJ, 525, 440

Martín, E. L., Montmerle, T., Gregorio-Hetem, J., \& Casanova, S. 1998, MNRAS, 300,733

Montmerle, T., Koch-Miramonde, L., Falgarone, E., \& Grindlay, J. E. 1983, ApJ, 269, 182

Muzerolle, J., Calvet, N., \& Hartmann, L. 2001, ApJ, 550, 944

Natta, A., Testi, L., Comerón, F., D’Antona, F., Baffa, C., Comoretto, G., \& Gennari, S. 2002, A\&A, 393, 597

Ozawa, H., Grosso, N., \& Montmerle, T. 2005, A\&A, 429, 963

Phelps, R. L., \& Barsony, N. 2004, AJ, 127, 420

Preibisch, T., Brown, A. G. A., Bridges, T., Guenther, E., \& Zinnecker, H. 2002, AJ, 124, 404

Preibisch, T., Guenther, E., \& Zinnecker, H. 2001, AJ, 121, 1040

Preibisch, T., \& Zinnecker, H. 1999, AJ, 117, 2381

Reipurth, B., \& Clarke, C. 2001, AJ, 122, 432

Reipurth, B., Pedrosa, A., \& Lago, M. T. V. T. 1996, A\&AS, 120, 229

Rydgren, A. E. 1980, AJ, 85, 438

Schmidt-Kaler, Th. 1982, in Landolt-Bornstein New Series, Numerical Data and Functional Relationships in Science and Technology, Group 4, Vol. 2b, ed. K. Schaffers \& H. H. Voigt (Springer: New York), 451

Sterzik, M. F., \& Durisen, R. H. 1995, A\&A, 304, L9

Struve, O., \& Rudkjøbing, M. 1949, ApJ, 109, 92

Torres-Dodgen, A. V., \& Weaver, W. B. 1993, PASP, 105, 693

Vrba, F. J., Strom, S. E., \& Strom, K. M. 1976, AJ, 81, 958

Vrba, F. J., Strom, S. E., Strom, K. M., \& Grasdalen, G. L. 1975, ApJ, 197, 77

Wilking, B. A., Greene, T. P., \& Meyer, M. R. 1999, AJ, 117, 469 (WGM99)

Wilking, B. A., \& Lada, C. J. 1983, ApJ, 274, 698

Wilking, B. A., Lada, C. J., \& Young, E. T. 1989, ApJ, 340, 823

Wilking, B. A., Schwartz, R. D., \& Blackwell, J. H. 1987, AJ, 94, 106

Wilking, B., Schwartz, R. D., Fanetti, T., \& Friel, E. 1997, PASP, 109, 549 